\title{
SDP Based Simulated Annealing on Bandwidth Reservation with Multi-Path Routing
}

Submitted by

\section{Baohua Zhang, B.Sc. in Applied Math}

\author{
A thesis submitted to \\ The Faculty of Graduate Studies and Research \\ in partial fulfillment of \\ the requirements for the degree of
}

\section{Master of Science (Information and Systems Science)}

\author{
School of Mathematics and Statistics \\ Ottawa-Carleton Institute of Mathematics and Statistics \\ Carleton University \\ Ottawa, Ontario, Canada K1S 5B6 \\ August, 2005 \\ (C) Copyright 2005, Baohua Zhang
}




$\begin{array}{ll}\begin{array}{l}\text { Library and } \\ \text { Archives Canada }\end{array} & \begin{array}{l}\text { Bibliothèque et } \\ \text { Archives Canada }\end{array} \\ \begin{array}{l}\text { Published Heritage } \\ \text { Branch }\end{array} & \begin{array}{l}\text { Direction du } \\ \text { Patrimoine de l'édition }\end{array} \\ \begin{array}{l}\text { 395 Wellington Street } \\ \text { Ottawa ON K1A ON4 }\end{array} & \begin{array}{l}\text { 395, rue Wellington } \\ \text { Ottawa ON K1A ON4 } \\ \text { Canada }\end{array}\end{array}$

Your file Votre référence

ISBN: 0-494-10149-0

Our file Notre référence

ISBN: 0-494-10149-0

NOTICE:

The author has granted a nonexclusive license allowing Library and Archives Canada to reproduce, publish, archive, preserve, conserve, communicate to the public by telecommunication or on the Internet, loan, distribute and sell theses worldwide, for commercial or noncommercial purposes, in microform, paper, electronic and/or any other formats.

The author retains copyright ownership and moral rights in this thesis. Neither the thesis nor substantial extracts from it may be printed or otherwise reproduced without the author's permission.
AVIS:

L'auteur a accordé une licence non exclusive permettant à la Bibliothèque et Archives Canada de reproduire, publier, archiver, sauvegarder, conserver, transmettre au public par télécommunication ou par l'Internet, prêter, distribuer et vendre des thèses partout dans le monde, à des fins commerciales ou autres, sur support microforme, papier, électronique et/ou autres formats.

L'auteur conserve la propriété du droit d'auteur et des droits moraux qui protège cette thèse. $\mathrm{Ni}$ la thèse ni des extraits substantiels de celle-ci ne doivent être imprimés ou autrement reproduits sans son autorisation.
In compliance with the Canadian

Privacy Act some supporting forms may have been removed from this thesis.

While these forms may be included in the document page count, their removal does not represent any loss of content from the thesis.
Conformément à la loi canadienne sur la protection de la vie privée, quelques formulaires secondaires ont été enlevés de cette thèse.

Bien que ces formulaires aient inclus dans la pagination, il n'y aura aucun contenu manquant.

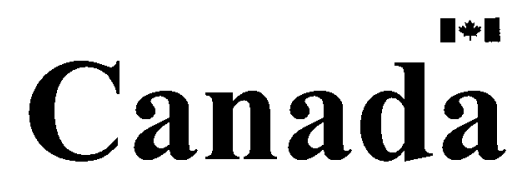




\section{Abstract}

Numerous routing schemes have been reported to improve network performances over

the years. Multi-path routing belongs to one of them and Multi Protocol Label Switching (MPLS) is an excellent platform for such routing. However, multi-path IP routing applied to the Internet has received little research attention because of the complexity of the problem and some intrinsic issues such as multi-objectives on path selection and traffic assignment on selected paths. In this thesis, an algorithm called Shortest Distance Path based Simulated Annealing (SDPSA) for finding optimal bandwidth reservation solutions for multi-path routing is developed to improve network performances. The algorithm, which employs the annealing method, is based on previous solutions to find the current sub-optimal solution for multi-path routing. Multiple objectives including balancing traffic load and minimizing network resource consumption are taken into consideration. The algorithm also integrates the interferences of multiple paths into IP routing optimization. Finally, the proposed algorithm is applied to a randomly generated network and the NSF network. The performance values are compared to a well-known multi-path routing algorithm-HSTwp. The simulation and comparison results show that the proposed SDPSA algorithm is efficient for the optimization of multi-path IP routing. 


\section{Acknowledgements}

I would like to thank my thesis supervisor-Prof. Changcheng Huang for his guidance and encouragement throughout the research. I would like to express my appreciation to him for his helpful advice and patience during the stage of my thesis study period at the Advanced Optical Network Laboratory.

I would like to express my appreciation to all my fellow graduate students at the Advanced Optical Network Laboratory at Carleton University for their kindness and friendship.

- iv - 
To my family

$-\mathrm{v}-$

Reproduced with permission of the copyright owner. Further reproduction prohibited without permission. 


\section{Table of Contents}

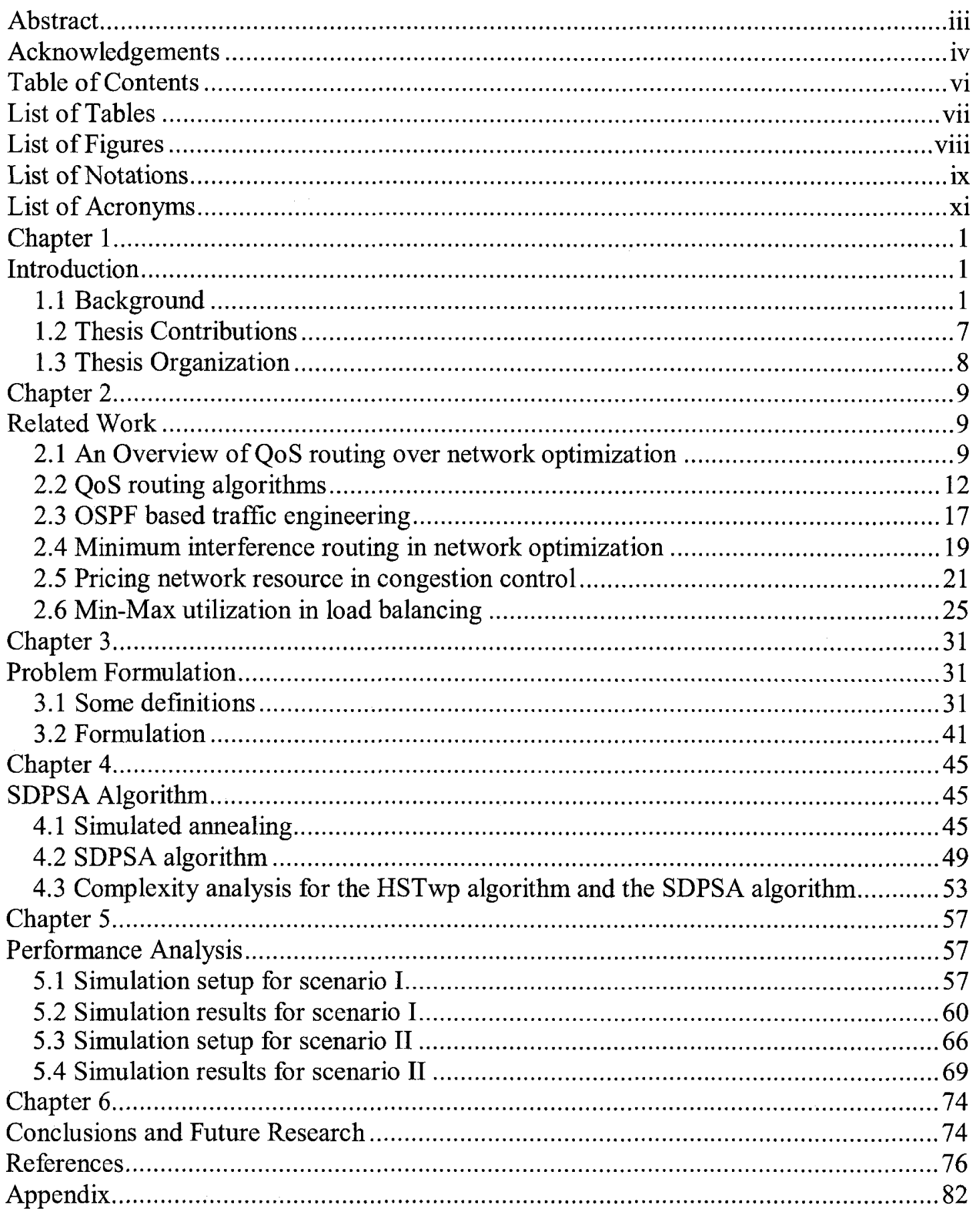

- vi - 


\section{List of Tables}

Table 2.1: The goals of different full information based QoS routing algorithms ..........13

Table 2.2: Pros and cons of flat pricing and usage sensitive pricing .............................23

Table 3.1: Candidate path set for four nodes network topology example.........................34

Table 3.2: Link capacity table of the example network ............................................. 37

Table 4.1: Complexity analysis for HSTwp algorithm and SDPSA algorithm ...............55

Table 5.1: Total resources consumed on all selected path ..........................................65

Table 5.2: Total resources consumed on all selected path for NSFNET ........................72

Table 5.3: Number of times in getting the suboptimal solution .................................. 73

- vii - 


\section{List of Figures}

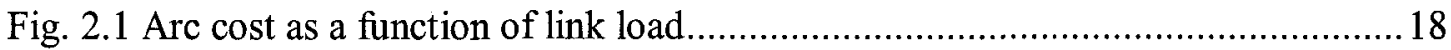

Fig. 2.2 Minimum Interference routing examples ................................................20

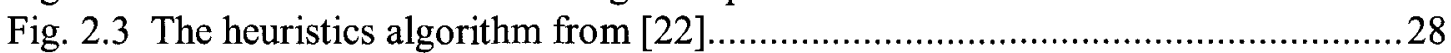

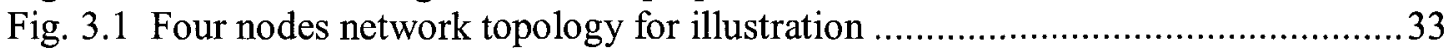

Fig. 3.2 SPS generator function flowchart....................................................... 40

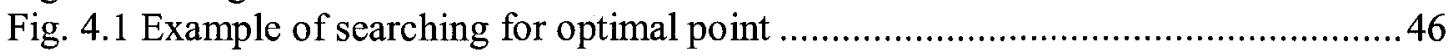

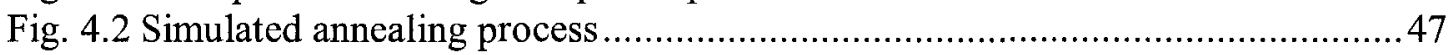

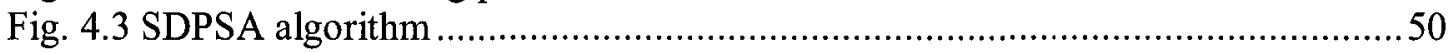

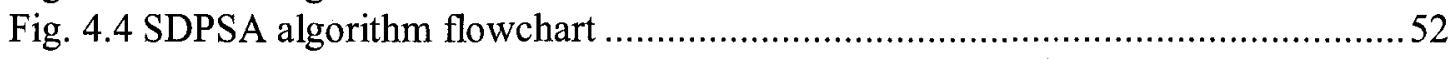

Fig. 5.1 Simulation Topology with 6 nodes and 10 bi-directional links ......................59

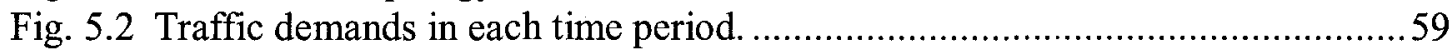

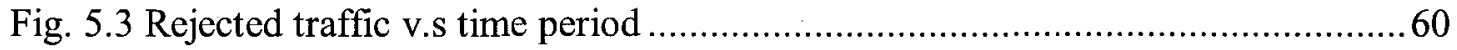

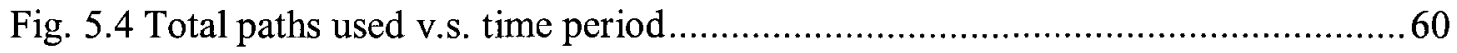

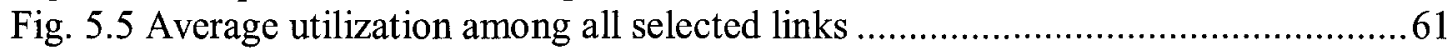

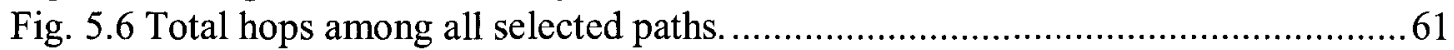

Fig. 5.7 Maximum utilization among all selected links ...........................................62

Fig. 5.8 Total interference links among all selected paths .....................................62

Fig. 5.9 NSFNET Topology with 14 nodes and 21 bi-directional links .......................66

Fig. 5.10 Traffic demands in each time period for NSFNET …................................67

Fig. 5.11 Total paths used v.s. time period for NSFNET ........................................69

Fig. 5.12 Average utilization among all selected links for NSFNET .........................69

Fig. 5.13 Total hops among all selected paths for NSFNET ..................................... 70

Fig. 5.14 Maximum utilization among all selected links for NSFNET ......................... 70

Fig. 5.15 Total resource used among all selected paths for NSFNET ...........................71

- viii - 


\section{List of Notations}

$G=(V, E)$

$V=\left\{v_{1}, v_{2}, \cdots, v_{N}\right\}$

$E=\left\{e_{1}, e_{2}, \cdots e_{M}\right\}$

$T_{d}(\tau)$

$(i, j)$

$r_{x y}^{i}$

$C_{x y}^{i}$

$C_{i j}$

$C^{\text {sps }}$

$U_{i j}$

$S_{i j}$

$S_{r_{x y}^{i}}$

$P\left(r_{x y}\right)$

$D\left(r_{x y}\right)$

$R$
The network graph has a set $V$ of nodes and a set $E$ of links.

The set of nodes in the network, $N$ represents the number of nodes.

all nodes are sorted as node $0,1, \ldots, N-1$

The set of edges in the network, $M$ represents the number of edges.

each edge has links $(i, j)$ and $(j, i)$ for traffic from both direction.

The traffic demands among all pairs of nodes in the network where

$\tau$ stands for various time periods

A link between node $i$ and node $j$

A path between node $x$ and node $y$ where $x, y$ belongs to set $V$

The bandwidth capacity of path $r_{x y}^{(i)}$

The bandwidth capacity of link $(i, j)$

The set of all SPS with all possible solution in accommodating given traffic demands.

The available bandwidth of link $(i, j)$

The total traffic assigned to link $(i, j)$

The traffic volume assigned to path $r_{x y}^{(i)}$

The path vector related to path $r_{x y}$.

The distance of path $r_{x y}$

Total network resource consumption

$-\mathrm{ix}$ - 
Total network resource consumption in HSTwp algorithm

$R_{S D P S A}$

Total network resource consumption in SDPSA algorithm

$-\mathrm{X}-$ 


\section{List of Acronyms}

\begin{tabular}{|c|c|}
\hline ATM & Asynchronous Transfer Mode \\
\hline $\mathrm{CAC}$ & Call Admission Control \\
\hline CPS & Candidate Path Set \\
\hline DiffServ & Differentiated Services \\
\hline DTMW & Double Threshold Moving Window \\
\hline EB & Effective Bandwidth \\
\hline ECMP & Equal Cost Multiple Path \\
\hline $\mathrm{EE}$ & Effective Envelops \\
\hline HSTsp & Heuristic algorithm of shortest path \\
\hline HSTwp & Heuristic algorithm of widest path \\
\hline IETF & Internet Engineering Task Force \\
\hline IntServ & Integrated Services \\
\hline ISP & Internet Service Provider \\
\hline LSP & Label Switched Path \\
\hline MPLS & Multi Protocol Label Switching \\
\hline NFS & National Science Foundation \\
\hline NFSNET & National Science Foundation Network \\
\hline NP & Non-deterministic Polynomial time \\
\hline PSR & Proportional Sticky Routing \\
\hline QoS & Quality of Service \\
\hline
\end{tabular}




$\begin{array}{ll}\text { RSVP } & \text { Resource Reservation Protocol } \\ \text { SA } & \text { Simulated Annealing } \\ \text { SDP } & \text { Shortest Distance Path } \\ \text { SDPSA } & \text { Shortest Distance Path based Simulated Annealing algorithm } \\ \text { SLA } & \text { Service Level Agreement } \\ \text { SPS } & \text { Selected Path Set } \\ \text { SWP } & \text { Shortest Widest Path } \\ \text { TSP } & \text { Traveling Salesman Problem } \\ \text { VLSI } & \text { Very Large Scale Integration } \\ \text { WSP } & \text { Widest Shortest Path }\end{array}$

- xii - 


\section{Chapter 1}

\section{Introduction}

\subsection{Background}

Current Internet routing utilizes the simple traffic forwarding method and provides only the 'best-effort' service with no guaranteed performance. With the rapid increase in the processing power of computers and the bandwidth of high-speed networks, new real-time multimedia applications such as Internet Telephony, Video Conferencing, and Virtual Private Networks are emerging. New applications have different Quality of Service (QoS) requirements such as bandwidth, delay, delay jitter, and packet loss. Current 'best effort' service over the Internet could not meet the QoS requirements for dynamic traffic demands $[1][2][3][4]$. Therefore, the ways to efficiently utilize the available network resources and satisfy different QoS requirements to improve network performance becomes an important issue.

The Internet Engineering Task Force (IETF) proposed different service models to meet the demands of QoS such as Integrated Services model (IntServ) [1], Differentiated Service model (DiffServ) [2], and Multi Protocol Label Switching (MPLS) [3]. The 
IntServ model is characterized by resource reservation. Using signaling protocol like Resource Reservation Protocol (RSVP), the resources are reserved before data are transmitted to ensure that the QoS requirements for each flow entering the network. The main problem for the IntServ model is the scalability problem as the amount of state information increases proportionally with the amount of flows, which in turn, overloads the core network.

In the DiffServ model, packets in different classes receive different services. Several different service classes can be created according to various QoS requirements. The amount of state information is proportional to the number of classes instead of that of flows. Therefore, the DiffServ model is more scalable than the IntServ model to meet the demands of QoS requirements. The IntServ model and DiffServ model are two standardized architectures for IP networks. MPLS is a technique that supports flow control, traffic engineering, load balancing, explicit routing, and tunneling. For the purpose of this thesis, the Shortest Distance Path based Simulated Annealing algorithm (SDPSA) was developed which is based on the integration of IntServ model with MPLS technique. In this thesis, we assume the effective bandwidth concept be used to estimate the bandwidths of flows as a basis for the development of this algorithm which is specifically designed for backbone networks. This is different from legacy IntServ model which requires deterministic guarantee under worst case scenario. Stochastic performance guarantee based on effective bandwidths allows more efficient resource usage than deterministic guarantee. 
Explicit routing seems to be a plausible solution for improving the quality of service requirements. Bandwidth reservation is a typical technique used for explicit routing to provide QoS services. By establishing explicit routes to meet bandwidth demands between each pair of nodes within the network, network performance can be optimized through different kinds of algorithms. Explicit resource management techniques are based on dividing all flows into different classes with various QoS level. Due to the nature of the routing protocol and forwarding methods, traffic tends to follow a shorter path with higher available bandwidth until the path becomes congested. Moreover, single path routing could result in traffic congestion which leads to poor overall network utilization [5] [6]. That is one of the reasons why congestion control becomes one of the major problems of network optimization.

Many congestion control algorithms reduce the traffic rate at the edge of the network based on the feedback provided by online traffic measurements. The online measurement technique uses different predictors such as Effective Bandwidth (EB) [7], Effective Envelops (EE) [8], or Double Threshold Moving Window (DTMW)[9] to predict the future traffic load.

The initial idea of effective bandwidth has been proposed in the context of connection admission control (CAC) for ATM networks. Effective bandwidth is a measure of resource usage, which adequately represents the trade-offs between different types of traffic streams, taking proper account of their varying statistical characteristics and QoS requirements [10]. From the perspective of CAC, effective bandwidth can be defined for 
an arbitrary collection of traffic sources as the minimum bandwidth needed at the multiplexer to support these sources together without violating the QoS requirements [11]. Moreover, besides CAC, the concept of effective bandwidth has also been applied to resource management and network dimensioning [12][13][14]. In our algorithm, we used the effective bandwidth concept for bandwidth resource reservation over different core networks. The assigned bandwidth to each selected path depends on the aggregate effective bandwidth estimation of traffic demands from the edge network.

There are other approaches for solving the network congestion problem by shifting the traffic to alternative paths [15][16]. Due to traffic shaping at the edge, traffic demands in the core network are usually stable over a given time period [17]. Given the topology of a network and capacity of the links, some online measurement approaches such as effective bandwidth approximation, can be used to estimate the available bandwidth over different links $[10][17][18]$.

To avoid uneven network utilization, numerous approaches with various objectives in network optimization have been proposed for efficiently utilizing the network resources such as $[19][20][21] ;$ Fortz's scheme adjusted link weights to optimize the network utilization [19]; Wang's scheme minimized the maximum utilization over links to improve the ability of accepting more future traffic [20]; Kodialam's scheme tried to establish a path with less interference by increasing the cost of critical links [21]; In [22], constrained multi-path routing scheme was formulated, and a heuristic algorithm called HSTwp was given by Lee et al. [22] to calculate the constrained multiple paths and their 
load splitting ratios. By splitting the traffic demands among multiple paths, it is possible to improve the overall network utilization through better load balancing schemes. However, splitting the traffic demands among multiple paths also raises new problems, for example, how to determine the number of paths and how to assign the volume of traffic to the selected paths in accommodating various traffic demands; how to adaptively accommodate the dynamic traffic demands by using multi-path routing algorithms, etc. Such issues require the candidate multi-path routing algorithms to be simple and scalable.

The goal of routing in communication networks is to set up a routing path or paths between source and destination nodes to forward user traffic in accordance with user's requirements and network restrictions. Typically, routing schemes consist of two components: information advertisement, and path search. Information advertisement represents the periodical update of routing information; path search represents the computation or examination performed to find a path or paths to achieve a given objective. The research interests in the routing of Internet traffic has recently shifted towards traffic engineering, that is, network performance optimization of operational networks. Multi-path routing is a load balancing technique in which the total load from a source to a destination is spatially distributed over several paths or channels. The general idea of multi-path routing technique is resource sharing. In our case, the shared resource is the link capacity. We will see that the proposed algorithm is an adaptive routing algorithm which allows efficient load balancing and network resource consumption. 
In addition, ways of efficiently utilizing the multi-path to transfer real time traffic is an important issue for addressing various QoS requirements especially with the dynamic traffic demands. However, there are few reports dealing with this problem. To overcome the inefficiency of current routing techniques, in this thesis, we will propose a new multipath routing algorithm called Shortest Distance Path based Simulated Annealing algorithm (SDPSA) to find a sub-optimal multi-path routing solution. This algorithm belongs to full information based algorithms in dealing with dynamic traffic demands.

SDPSA algorithm proposed here is a simple adaptive routing algorithm which allows efficient load balancing and network resource consumption. Our objective is to find a set of paths that can accommodate traffic demands with as little consumption of network resources as possible. At the same time, network load balancing is considered to reduce network congestions. Through simulated annealing, the summation of distances of selected path set (SPS) is minimized. Starting from the previous solution as initial annealing point, the SDPSA algorithm is more efficient in path set re-selection as traffic demands change dynamically. 


\subsection{Thesis Contributions}

The contributions in this research are briefly summarized as follows:

- Combined two objectives in IP routing: shortest path first and load balancing. In such a way, our solution can balance the network traffic and consumes as little network resources as possible at the same time. Taking the minimum sum of shortest distance paths over selected path set makes it possible to combine those two objectives.

- Proposed a new algorithm, the SDPSA algorithm, which applies simulated annealing method to the multi-path routing problem and gives a sub-optimal solution to multi-path routing problem. Given the topology and capacity of a core network, SDPSA algorithm tries to find a multi-path routing solution for dynamic traffic demands and consume as little network resources as possible while balancing traffic loads across the network at the same time.

- Implemented and conducted simulations to verify the performance of SDPSA. We applied our algorithm to two topologies: One is randomly generated; the other is the well-known NSF network. The results are compared to a well-known multipath routing algorithm, HSTwp. The simulation and comparison results show that the proposed SDPSA algorithm is feasible and efficient for the optimization of multi-path IP routing. 


\subsection{Thesis Organization}

The remaining chapters of the thesis are organized as follows.

- Chapter 2: Introduces works related to QoS routing. Different approaches with different objectives on network optimization will be discussed in this chapter.

- Chapter 3: Gives some definitions and notations, we formulate a network optimization problem and develop the SDPSA algorithm to solve the problem; a detailed description of objectives related to the formulation problem is given afterwards.

- Chapter 4: Following a brief description of the background of simulated annealing, the SDPSA algorithm is developed.

- Chapter 5: Applies SDPSA algorithm to a randomly generated network example and NSF network. The simulation results are analyzed and compared with a well-known multi-path routing algorithm - HSTwp.

- Chapter 6: Summarizes our work and identifies some directions for future research. 


\section{Chapter 2}

\section{Related Work}

\subsection{An Overview of QoS routing over network}

\section{optimization}

In an IP network, resource management techniques are either over provisioning or explicit resources management. Over provisioning does not allow bottleneck to appear in a communication system. This method is used because the new transmission technologies provide high bandwidth. However, it seems that the usage of bandwidth grows as fast as the increase of bandwidth. Moreover, this technique can be less profitable for ISP because all users have the same QoS level treatment.

IETF defines service level agreement (SLA) as "a service contract between a customer and a service provider that specifies the forwarding services a customer should receive" [2]. Currently, all major routers/switch vendors like Cisco, Juniper, and Lucent provide some QoS mechanisms in their high-end products, thus even if bandwidth eventually becomes cheap and abundant. This is not likely to occur soon. 
Depending on the ways of making a forwarding decision, there are two different kinds of QoS routing services. The first one is connection-oriented which sets up the routing path before the transfer of data. The second one is connectionless-oriented where each packet has enough information (destination address) to enable any switch to decide how to reach its destination. In the connectionless-oriented service, the notions of a flow of packets such as a micro flow are adopted to bring in connection-oriented behavior to provide QoS services.

A QoS routing algorithm works as follows: for each arrival request, the algorithm either selects a route that satisfies the QoS requirements or rejects it. Usually, more than one route is available; the network performance depends on the decisions that the QoS routing algorithm makes.

For the QoS routing algorithms, many of the proposed schemes focus on how to find a feasible path to satisfy the QoS requirement. These algorithms are usually based on the assumption that the network state information can be obtained through certain methods.

For different QoS requirement from users, whether based on traffic profile or estimation, a feasible path or multi-path can be found to achieve the QoS requirement and, at the same time, reduce the cost by balancing the load and congestion. Those schemes are usually focused on link or path utilization, blocking probability, loss or delay metrics. 
There are different methods proposed to solve the problem of minimizing resource conservation and, at the same time, to balance the load over all links in the network [4]. The equal-cost-multi-path option of OSPF [4] and IS-IS Optimized multi-path [23] is an improvement in distribution load to several shortest paths. Unfortunately, ECMP is not helpful if there is only one shortest path. Manually configuration of the cost of links to evenly distribute the traffic is another attractive scheme. However, it is difficult to deal with complex ISP networks [24].

The traffic engineering and network optimization schemes usually deal with network traffic management because of different objectives. Based on related objectives and some constraints, an optimal solution or sometimes a sub-optimal solution can be calculated depending on the completeness of various algorithms. Fortz proposed Open Shortest Path First based traffic engineering scheme in [19]. Wang suggested a scheme based on minimum maximum utilization as their objective [20]. The authors in [21] argue that minimum interference routing algorithm improves the network performance through minimizing the interferences of links in selecting routing paths. Many others suggested pricing network recourses instead of flat rate price to solve congestion problem in order to meet different kinds of QoS requirements [25][26][27][28]. 


\subsection{QoS routing algorithms}

QoS routing selects feasible paths that meet a set of QoS constraints and selects an efficient path that achieves higher network throughput if the feasible path is more than one. A feasible path is one that has sufficient unused resources to satisfy the QoS constraints of a connection. QoS routing consists of two tasks. The first one is to collect the state information and keep it up to date. The second one is to find a feasible path for a new connection based on the collected information. QoS routing algorithms use two different objective functions to optimize network performance. For the purpose of minimizing cost, the shortest path is typically selected; for the purposes of load balancing, the least loaded path is selected. The combination of the two is challenging to achieve [29][30].

To improve the quality of service requirements, it seems that explicit routing is a more capable solution than destination based routing. By setting up explicit routes to meet bandwidth demands between edge nodes of the network, network performance can be optimized through different kinds of algorithms. Explicit resource management techniques are based on dividing all flows into different classes with various QoS levels. It requires traffic control mechanisms such as admission control, policing, classification, and scheduling. 
From the network state metrics point of view, there are full information-based QoS routing algorithms, also called globalized QoS routing, and partial information-based QoS routing referred as localized QoS routing. Full information-based QoS routing algorithms require full network state information to be exchanged periodically. Examples of the full information-based QoS routing algorithms are discussed in [29][30][31][32]. In contrast, partial information-based QoS routing algorithm uses partial information collected locally, and it does not have knowledge about the state of the rest of the network [33].

Table 2.1: The goals of different full information based QoS routing algorithms

\begin{tabular}{|l|l|}
\hline Algorithms & The goal of QoS routing algorithms \\
\hline SWP & Find a path with Maximum bottleneck bandwidth \\
\hline SDP & Find a path with the shortest distance \\
\hline WSP & Find a feasible path with minimum hop counts \\
\hline
\end{tabular}

There are several examples of full information based QoS routing algorithms like shortest widest path (SWP), shortest distance path (SDP), widest shortest path (WSP) $[24][34][35]$. Each of them has different objectives when choosing the "best path" (Table 2.1). 
SWP algorithm first tries to find the widest path with the maximum bottleneck bandwidth, and then minimizes hop counts. SWP algorithm calculates the shortest widest path. The aim is to find a path with maximum bottleneck bandwidth (a widest path), and when there are more than one widest path, the one with shortest propagation delay is chosen. If several such kinds of paths have the same hop counts and same bandwidth, one is randomly chosen. SWP optimizes first bandwidth, after that hop counts.

WSP algorithm searches for the minimal hop counts first, and then the one with the maximum bottleneck bandwidth is chosen. WSP algorithm calculates widest shortest path. It searches for a path with minimal hop counts, if there is more than one shortest path exist, the one with maximum bottleneck bandwidth is chosen. If several such kinds of paths have the same hop counts and the same bandwidth, one is randomly chosen. WSP first optimizes hop count, and then bandwidth.

SDP algorithm tries to find a feasible path with the shortest distance and strike a balance by using distance function. Suppose path $r$ has $k$ links; $C_{j}$ is the available bandwidth of link $j$ and $j$ is from 1 to $k$. The distance of path $r$ is defined in the formula below:

$D(r)=\left(1 / C_{1}\right)+\left(1 / C_{2}\right)+\ldots+\left(1 / C_{k}\right)$

Full information-based QoS routing can achieve optimal solution if the state information is accurate. The frequent state updates create much overhead and this may lead to congestion especially when the network is large. Through locally collected flow statistics 
such as flow arrival rates, flow departure rates, or flow blocking probabilities, source router can perform adaptive proportioning of flows among a set of candidate paths based on this local information [34].

There are some schemes of partial information-based QoS routing that can address the scalability problem like [15] [33]. In [33], proportional sticky routing scheme attempts to use the minimization technique of equalized blocking rate of candidate paths based on the Erlang loss Formula [36]. In proportional sticky routing scheme (PSR), only edge routers need to keep track of the route level statistics and recomputed proportions after every observation period. The PSR scheme can be viewed to operate in two stages: the first one is proportional flow routing, and the other one is computation of flow proportions.

In [15], the MATE algorithm is discussed. The main goal of the MATE algorithm is to avoid network congestion by adaptively balancing the load among multiple paths based on measurement and analysis of path congestion using the state information between a pair of Ingress and Egress pair. Partial information-based QoS scheme are used to solve the scalability problem. However, it leads to non-optimal allocation of resources because of the lack of global view of network state information.

Another classification of QoS routing algorithms divide them into static state QoS routing algorithms and dynamic state QoS routing algorithms. The static state routing algorithms are able to find the 'best' path (or paths) route without knowing either past or the future 
traffic demand. On the other hand, the dynamic state routing algorithm finds new routing path or paths with the dynamic change of traffic demands.

The SWP algorithm, WSP algorithm, and SDP algorithms mentioned earlier all belongs to the static state routing algorithm. Minimizing the overall cost through optimized weight assignments adjustment proposed by Fortz [37] also belongs to static state routing algorithm.

The proportional sticky routing scheme in [33], the MATE algorithm in [15], the MinMax utilization proposed by Lee [22], and the SDPSA algorithm presented in this thesis belong to the dynamic state routing algorithm. Those algorithms usually generate new path (paths) or shifting some traffic from high utilized path to lower utilized paths to accommodate dynamic traffic demands for improving the network performance. 


\subsection{OSPF based traffic engineering}

In [19] and [37], Fortz et al. use OSPF/IS-IS routing with optimized weight settings in order to improve the network quality of service. The optimization objective is $\sum_{l \in \text { links }} f\left(\rho_{l}\right)$, where $f\left(\rho_{l}\right)$ is a piecewise linear and convex function over link

utilization $\rho_{l}$. As $\rho_{l}$ approaches one, the slope of $f$ increases dramatically and causes the optimization process to avoid link overload (Fig. 2.1)

Their simulation result is mainly based on AT\&T's IP backbone with 90 nodes and 274 links. It has been shown that by properly setting the weights in an OSPF/IS-IS network, significantly better network throughput and performance can be achieved compared with the simple default weight settings recommended by Cisco's defaults inverse-capacityweights. The local search heuristics explores the neighborhood of an initial weight vector by modifying individual link metric setting in a greedy fashion [19]. 


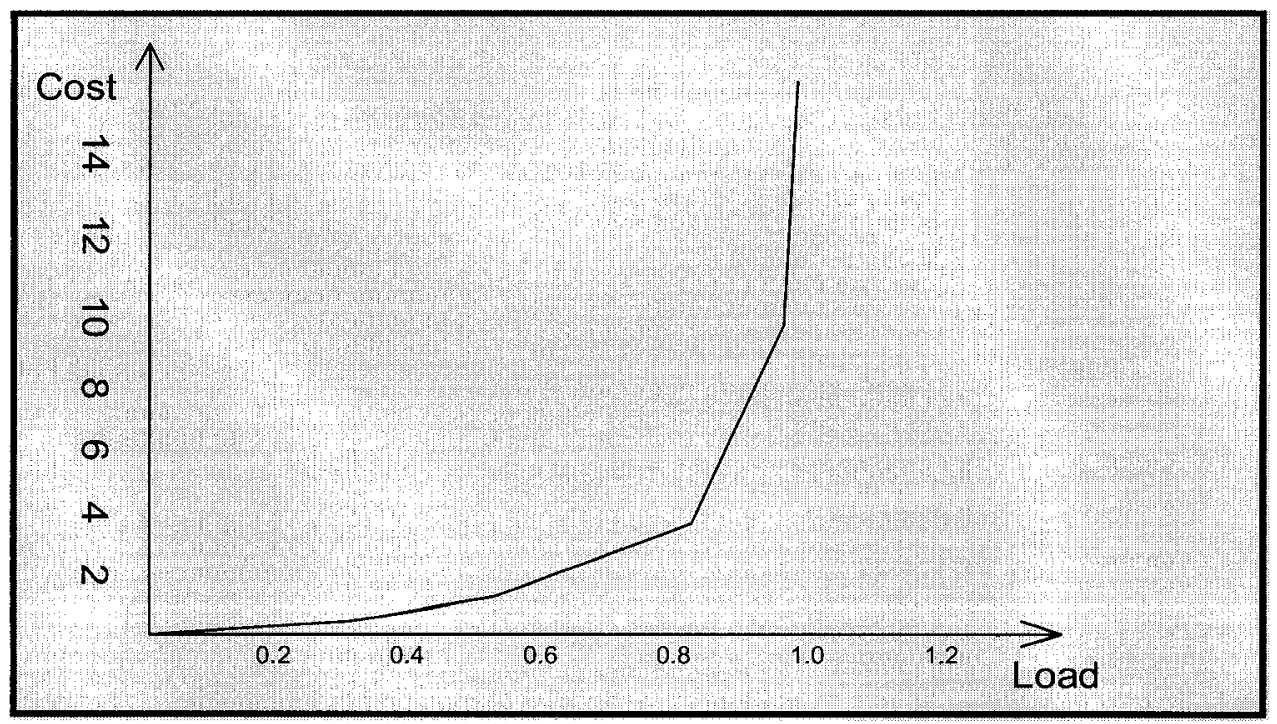

Fig. 2.1 Arc cost as a function of link load

The limitation of this research is that the stated optimizations were done offline based on static traffic loads in the network. Because the weight settings of the links are static, the routing algorithms are not adaptive to real network traffic loads, which are very dynamic. Since the algorithms need the traffic statistics and will do the optimization offline, they are hard to implement in real networks. One of the reasons is that the link cost depending on the link capacity and the traffic demands going through the link. With the dynamic traffic demands, the link cost for all links will be dynamic too. This makes the lack of real-time information during offline optimization in real network. Moreover, after the optimized weight calculated, the traffic demand might have changed already. 


\subsection{Minimum interference routing in network}

\section{optimization}

Lakshman et al. [21] provide a different approach in solving routing optimization and capacity assignment problem in the field of traffic engineering. Given the network topology and each individual link and node capacity, there can be two categories of objectives. The first one is maximizing the future traffic throughput we addressed in last subsection and the second one is optimizing the quality of service. For certain predicted or measured traffic demands, finding a routing pattern to optimize the network is the objective of the scheme.

Minimum interference routing scheme is defined in MPLS domains for achieving the objectives of traffic engineering. The authors in [21] assume that the LSP setup requests arrive on by one.

The objective of this scheme is to maximize the acceptance ratio of future arrival requests by trying to avoid the critical links as much as possible. Critical links is defined as the following: whenever an LSP is routed over these links the maximum flow values ${ }^{1}$ of one or more ingress-egress pairs will decrease. As shown in Fig. 2.2, after the setup of the

\footnotetext{
${ }^{1}$ The maximum-flow value refers to the determined maximum amount of flow that can be sent from a source node to a sink node in a directed network with the given link capacities [39].
} 
LSPs between S1,D1 and S2, D2, link 7-8 is a critical link when we setup LSP between S3,D3 even though path 1-7-8-5 has less hop count than 1-2-3-4-5. Setting path 1-7-8-5 as an LSP can decrease the max-flow values of ingress-egress pair S1, D1 and S2, D2 because of link 7-8. This link is the critical link. Detailed information can be found in [21].

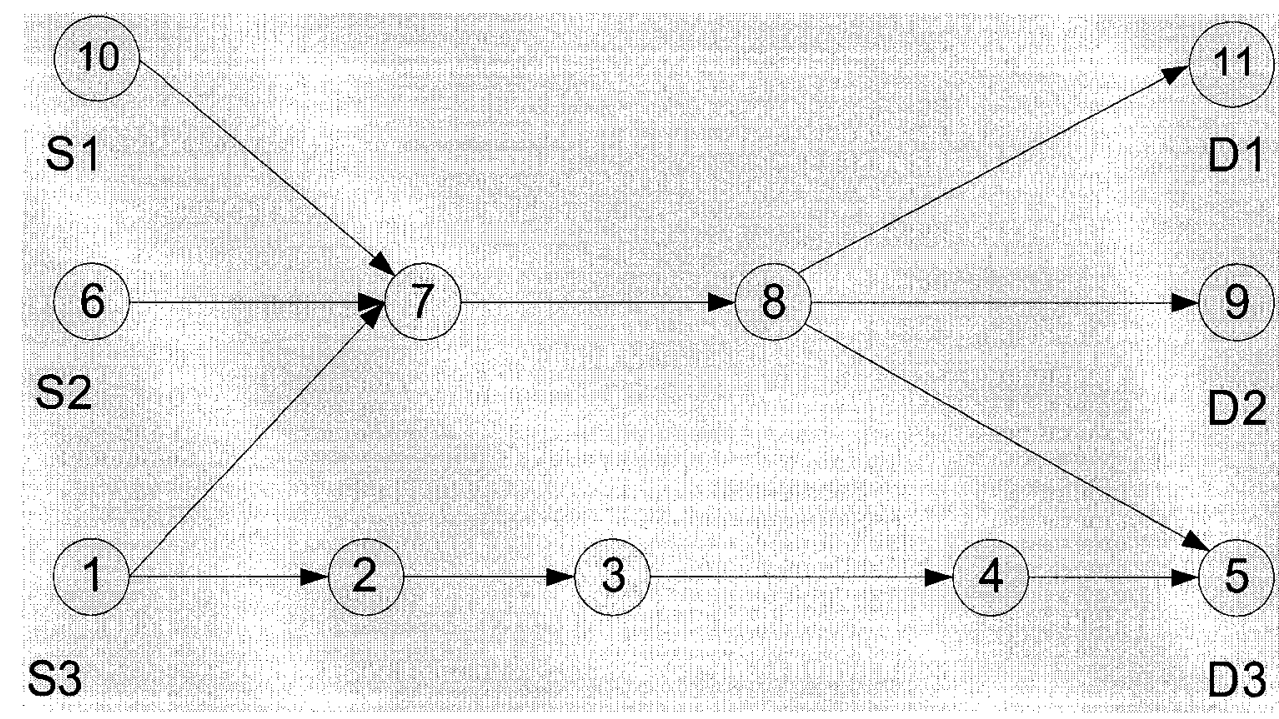

Fig. 2.2 Minimum Interference routing examples

This scheme can accommodate future new LSP setup requests by choosing the less utilized path. However, in a relative large network we can imagine that the computation for the critical link could be very expensive. Moreover, the frequencies of critical link identification and the intervals of update also need to be considered. 


\subsection{Pricing network resource in congestion control}

The effects of pricing network resource with traffic management have been examined by different researchers in network communication field in the recent years. There is considerable amount of research discussing the use of different schemes of pricing policy in order to improve overall network utilization and network performance[25][26][27][28]. Some papers also discuss how the pricing scheme would encourage users to use the network [25][27]. However, there are ideas for preserving the simplicity of the network by using the flat rate pricing scheme as used currently with the Internet. ${ }^{2}$ Flat rate pricing refers to a tariff that is independent from the amount of traffic produced. Many Internet service providers charge users a monthly fee regardless of the amount of traffic is generated.

Usage sensitive pricing, on the other hand, means that prices are a function of the amount of traffic that actually flows through a connection. Whether to use flat rates or usage sensitive pricing becomes a heated debate [25][27]. In the following part of this section, we will give an example of pricing based resource allocation scheme to satisfy various QoS requirements.

\footnotetext{
${ }^{2}$ Currently the Internet Service Provides, like Rogers and Bell, are still using the flat rate method to charge users.
} 
In paper [25], Jordan discussed the role of pricing in connection establishment process. Unlike others, Jordan took both bandwidth and buffer into consideration. To maximize the total utility of all users in the network under given loss constraints, this scheme provides a guidance of developing feedback algorithms in adjusting buffer and bandwidth in terms of various QoS level. Assuming there are $m$ Classes of virtual circuit traffic, the problem can be defined in the formula below:

$$
\begin{aligned}
& \max _{B W_{j l} B F_{j l}} \sum_{j=1}^{m} U_{j}\left(L_{j}, N_{j}\right), \\
& S . T . \\
& \sum_{j=1}^{m} B W_{j l} \leq B W_{l}, \sum_{j=1}^{m} B F_{j l} \leq B F_{l}, \\
& \sum_{l=1}^{n_{j}} D_{j l} \leq D_{j}, B W_{j l} \geq 0, B F_{j l} \geq 0 .
\end{aligned}
$$

$B W_{j l}, B F_{j l}$ : the bandwidth and buffer reserved by class $j$ traffic before link $l$

$B W_{l}, B F_{l}$ : the total available buffer and bandwidth over link $l$

$D_{j l}=B F_{j l} / B W_{j l}$ : the maximum delay for class $j$ traffic at link $l$.

$D_{j}$ : total upper end to end delay bound for class $j$.

$n_{j}$ : number of links on virtual path for class $j$ traffic

$N_{j}$ : assuming there are $N_{j}$ independent and identically distributed flows within class $j$

$L_{j l}: L_{j l}=g_{j}\left(B W_{j l}, B F_{j l}, N_{j}\right)$ denotes the probability of loss of class $j$ on link $l$ as a function of the bandwidth allocated to the class, the buffer allocated to the class, and the number of flows $N_{j}$

$L_{j}: L_{j}=\sum_{l=1}^{n_{j}} L_{j l}$ total end to end loss probability for class $j$ 
$U_{j}\left(L_{j}, N_{j}\right)$ : class $j$ gets $U_{j}\left(L_{j}, N_{j}\right)$ satisfaction from supporting $N_{j}$ sources with a probability loss of $L_{j}$.

As we can see in $L_{j l}=g_{j}\left(B W_{j l}, B F_{j l}, N_{j}\right)$, the function $g$ of loss probability for each individual class $j$ is difficult to get. In connection-oriented networks as discussed in this thesis, e.g., MPLS and ATM networks, the total end to end loss probability $L_{j}$ over a path is approximately equal to the sum of loss probability over all links along the path [25]. The author also assumes that the distributed flows within class $j$ are independent and identical, which might not always be the case of some real networks.

Table 2.2: Pros and cons of flat pricing and usage sensitive pricing

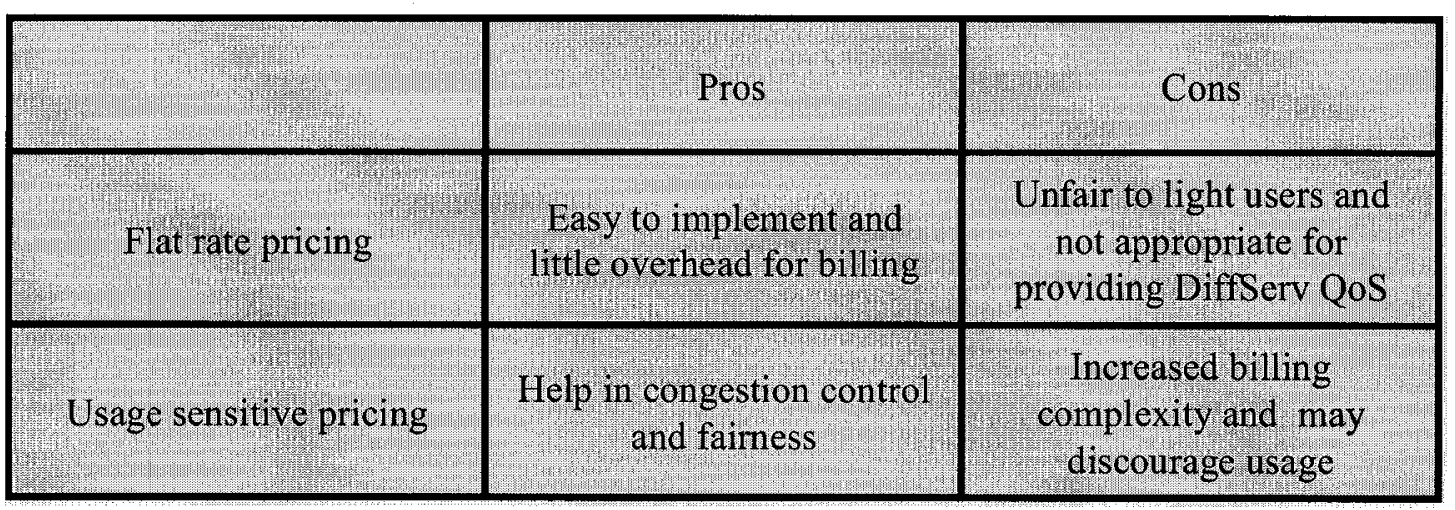

The network pricing has been controversial topic over years, flat rate means users pay the same price every month or year regardless of the traffic they generate. Usage sensitive pricing charges users based on the basis of their use. Flat rate is simple for billing purposes and encourages users to use the network as much as possible which leads to network congestion. Many users do not like to use the sensitive pricing scheme because 
it can be inconvenient and as a result discourage the use of the network. However, usage sensitive pricing scheme helps to control network congestion by increasing the link price when the load is heavy and decreasing the price when the load is reduced. Usage sensitive pricing scheme achieves fairness and satisfies different QoS requirements on the part of users. The pros and cons of flat pricing and usage sensitive pricing are outlined in Table 2.2. 


\subsection{Min-Max utilization in load balancing}

In order to be more competitive in commercial market, ISPs must constantly improve the QoS by reducing delay, packet losses and increasing throughput experienced by end users. ISPs have to meet certain performance objectives and, at the same time, maintain a high level of resource utilization to maximize the return of their investment in network assets. Minimizing network congestion is an important objective. There are two typical factors leading to network congestion: first, traffic demands far exceeding the network capabilities and second, having poor traffic distribution while some links in the network are overloaded and others are underutilized. To address the poor traffic distribution over the network, minimizing the maximum of link utilization is an important optimization objective. When the maximum of link utilizations over a network is minimized, the percentages of residual bandwidths over all network links are maximized, which is likely to accommodate more future traffic demands.

Over the years the multiple-path routing scheme has been considered to improve network performance. Since there are hundreds of flows in the intermediate node, flows must be handled in an aggregated manner because of the scalability problem. If flows are established only along the shortest paths, bandwidths on those paths will soon be consumed leading to high call-blocking rates. Exploring multiple paths is the key for 
solving the performance problem. On the other hand, multiple paths routing also brings many questions such as how to select paths and how to assign traffic to selected paths.

In [20][38] Wang et al. solve the routing optimization problem with the objective of minimizing the maximum link utilization. They propose an algorithm that takes the solution of the linear multi-commodity flow problem as the starting point. The maximum-flow problem involves multiple commodities, in which each commodity has an associated demand and source-sink pairs [39]. As this solution usually contains demands which are split over multiple paths, a subsequent algorithm reroutes all bifurcated flows in order to obtain only one LSP per end-to-end demand.

They model the backbone network as a set of nodes connected by links with fixed capacities. The network is represented as a directed graph $G(V, E) . \quad V$ is the set of nodes and $E$ is the set of links. For each link $(i, j)$ in $E$, let $C_{i j}$ to be the capacity of the link. Let $K$ represents the set of traffic demands between a pair of edge nodes. For each $k \in K$, let $d_{k}, s_{k}, t_{k}$ be the bandwidth demand, source, and destination node. Let $X_{i, j}^{k}$ be the percentage of $k$ 's bandwidth demand provided by link $(i, j)$. Let $\alpha$ be the maximum of link utilization among all the links. 


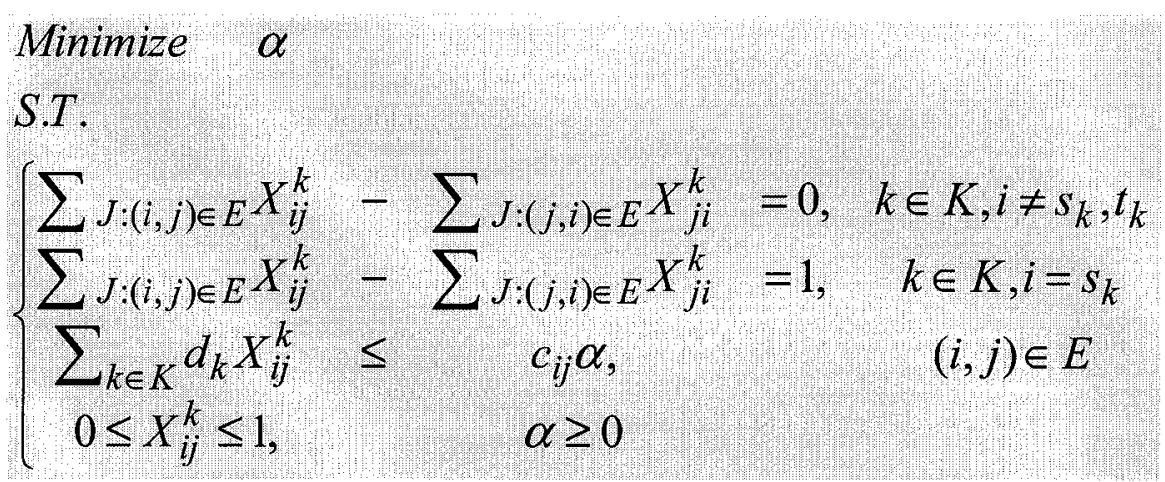

By minimizing the maximum utilization, the network load balance is achieved, and there is an increase in accommodating the future incoming traffic. This algorithm is based on the assumption that the traffic demands between each Ingress and Egress pairs and the network metric of link capacity are known. This assumption requires very accurate online measurements. The complexity of computation is an issue which has to be considered. There are some extensions to this work and a constrained multi-path routing in MPLS by Lee et al. [22] is one of them.

In paper [22], Lee et al. claim that there might be unnecessarily long paths because the objective functions of minimizing the maximum utilization only [20]. They therefore formulate the objective as a constrained multi-path routing problem that is a variation of minimizing maximum utilization problem. The constraint is that each path needs to satisfy a maximum hop count in multi-path routing. 
Heuristic: Finding constrained multiple paths and their load splitting ratios

- Set $\alpha$ to be current maximum link utilization in the network;

- $d_{k}$ is the requested traffic demand from $s_{k}$ to $t_{k}$;

- Convert $G$ to $L_{k}$-hop constrained graph, $G^{\prime}$;

- Find a set of $M$ shortest (or widest) paths from $s_{k}$ to $t_{k_{j}}$ $P=p_{i}, i=1, \ldots, M$;

- Set $\alpha_{M}$ to be the maximum link utilization of $P$;

if $\left(\alpha_{M}<\alpha\right)$ while ( $d_{k}>0$ and $P$ is not empty)

- Set $p$ to be the minimum hop path in $P$;

- Assign $d_{k}$ to the path, $p$, until $\alpha_{M}$ is less than $\alpha$;

- Delete $p$ from P;

- Update $d_{k}$ and $\alpha_{M}$;

endwhile

endif

while $\left(d_{k}>0\right)$

- Assign remaining $d_{k}$ to $M$ paths in proportion to the available link capacity;

endwhile

Fig. 2.3 The heuristics algorithm from [22]

A heuristic algorithm, the HSTsp algorithm (or the HSTwp algorithm) is proposed in [22] to find constrained multiple paths and their load splitting ratios. In the HSTsp algorithm, the $K$ shortest path algorithm is used for finding $K$ multiple paths. By contrast, the HSTwp algorithm tries to find $K$ widest paths by choosing paths with the maximum reserveable bandwidth among all the paths from a source to a destination. The detailed algorithm is described in Fig. 2.3

The HSTsp or HSTwp algorithm contains four parts: 
- Selecting one of the LSP connection requests: all LSP requests will be sorted according to the given priority or the administrative policy such as the maximum of the traffic demands.

- Converting given network to a hop-count constrained graph for eliminating additional cycles.

- Finding $K$ multiple paths: the HSTsp algorithm uses $K$ shortest paths algorithm ${ }^{3}$ while the HSTwp algorithm selects paths based on their maximum reserve-able bandwidths among all the paths from a source to a destination.

- Calculating load-splitting ratio: the amount of traffic, $d_{k}$, is assigned to the paths found in previous step. If the maximum of link utilizations on the $K$ paths is less than the current bottleneck link utilization, the traffic demands are split to minimize the amount of used total resources. Otherwise, all paths related to this link are eliminated from the $K$ multiple paths.

The HSTsp or HSTwp algorithm proposed by Lee et al. [22] helps to find a near optimal solution of minimizing the most congested link. In addition, the algorithm adds the maximum hop constraint to reduce the loop in the selected multiple paths. However, the $K$ multiple paths found by the HSTsp or HSTwp algorithm might not be the paths that could minimize the total network resource consumption.

\footnotetext{
${ }^{3}$ Yen's K shortest path algorithm was proposed in [44]. This algorithm is one of the best algorithms to find $\mathrm{K}$ shortest loop-less path [45]. The whole algorithm using a $\mathrm{O}\left(\mathrm{N}^{2}\right)$ Dijkstra algorithm to calculate the shortest path, and the approximate upper bound of complexity is at $\mathrm{O}\left(\mathrm{N}^{3}\right)$ level, where $\mathrm{N}$ is the number of nodes.
} 
Moreover, minimizing maximum utilization is only one approach to balance the load in the network. There might be other methods that can balance the load and minimize the total network utilization at the same time. Motivated by this, we re-formulate the multipath routing problem on bandwidth reservation (Chapter 3) and provided an algorithm (Chapter 4) to solve this problem. 


\section{Chapter 3}

\section{Problem Formulation}

A backbone network can be modeled as a bi-directional graph $G(V, E)$. Nodes or vertices $\{V\}$ represent switches, routers, or hosts; edges $\{E\}$ represent communication edges, $N=|\eta|$ and $M=|E|$ denote the number of vertices and edges respectively. For each edge in $E$, there will be two links for both traffic directions, they are expressed as $(i, j)$ and $(j, i)$. Throughout this thesis, $(i, j)$ will be used to denote a link from node $i$ to node $j$ and $r_{x y}$ is used to denote a path from ingress node $x$ to egress node $y$ unless specified otherwise.

\subsection{Some definitions}

Traffic demand matrix: For each link $(i, j)$ in $E$, where $i$ and $j$ are network nodes, let $C_{i j}$ be the capacity of the link. Let $T_{d}(\tau)$ represent the set of all traffic demands among all pairs of ingress and egress routers over a MPLS network at time $\tau$. The traffic demand matrix can be written as the matrix $T_{d}(\tau)$ below; where $\tau$ stands for each time period. For example, time period $\tau_{1}$ stands for time $T_{0}$ to $T_{1}$ and $\tau_{2}$ stands for time $T_{1}$ to $T_{2}$, and so on. 


$$
T_{d}(\tau)=\left[\begin{array}{ccccc}
0 & t_{0,1}(\tau) & \ldots & t_{0, N-2}(\tau) & t_{0, N-1}(\tau) \\
t_{1,0}(\tau) & 0 & \ldots & t_{1, N-2}(\tau) & t_{1, N-1}(\tau) \\
\ldots & \ldots & 0 & \ldots & \ldots \\
t_{N-2,0}(\tau) & t_{N-2,1}(\tau) & \ldots & 0 & t_{N-2, N-1}(\tau) \\
t_{N-1,0}(\tau) & t_{N-1,1}(\tau) & \ldots & t_{N-1, N-2}(\tau) & 0
\end{array}\right]
$$

Where $t_{i, j}(\tau)$ stands for traffic demands from node $i$ to node $j$ and $t_{i, j}(\tau)$ is piecewise constant in each time period $\tau .(0 \leq i, j \leq N-1)$

As we can see, we have a total of $N^{*}(N-1)$ pairs of nodes in the network. We assume here that there is a good enough online measurement algorithms that can predict the future traffic relatively accurately. For example, we can use the aggregation of effective bandwidth from edge network. This way, we can obtain relatively accurate traffic matrix $T_{d}(\tau)$ indicating the future traffic demands in the next time period after $\tau$. The time period can be measured in hours or days. Within each of these time periods, the traffic demand matrix is fixed.

This assumption is reasonable according to the previously reported work [40]. As mentioned in [40], traffic profile can be taken from the service level agreements, created by rule-of-thumb or any other mechanisms suitable to the network operator. Fig. 3.1 is a four node network topology facilitating understanding the definitions used in this chapter. 
In Fig. 3.1, we have a total of $4 * 3$ pairs of nodes such as pair $(0,1)$, pair $(1,0)$, pair( $(0,2)$, pair(2,0), pair(0,3), pair(3,0), pair(1,2), pair(2,1), pair(1,3), pair(3,1), pair(2,3), and pair $(3,2)$. For the reason of simplicity, here we use the link id number as the notation to identify each individual link (see Fig. 3.1), functioning the same as the node pair did before. Since each link has two directions, we use transpose ' to identify the other direction of the link, e.g., the number in (1'2) indicates a link starts from node 2 , traverses node 1 , and ends in node 3, where link 1 refers to the link from node 1 to 2 . Let us assume that for the first period of time, the traffic demand matrix $T_{d}\left(\tau_{1}\right)$ is as following:

$$
T_{d}\left(\tau_{1}\right)=\left[\begin{array}{cccc}
0 & 0 & 0 & 450 \\
0 & 0 & 0 & 0 \\
0 & 350 & 0 & 0 \\
0 & 0 & 0 & 0
\end{array}\right] \quad \text { Where } t_{0,3}\left(\tau_{1}\right)=450 ; \quad t_{2,1}\left(\tau_{1}\right)=350
$$

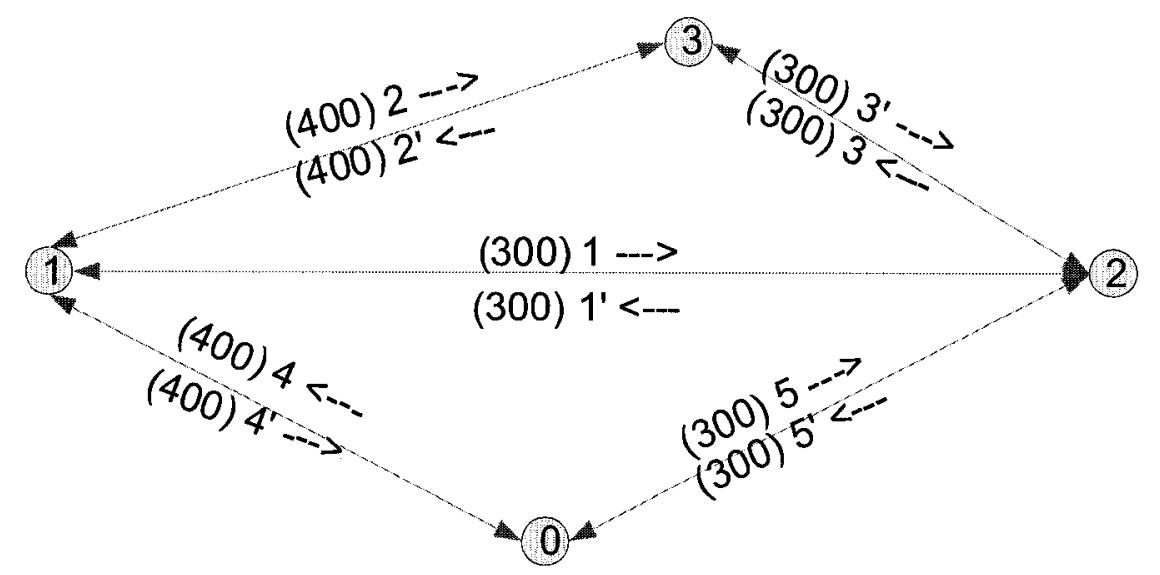
Legend $\quad(C) I \quad$ the link with ID $=i$ has the capacity of $C$ and the direction is as the arrow shows

Fig. 3.1 Four nodes network topology for illustration

We define the following concepts: 
Candidate Path Set (CPS): All paths between an ingress and egress pair are candidate paths for this ingress-egress pair. A Candidate Path Set (CPS) is the set of all paths for all ingress-egress pairs in a given topology.

In Fig. 3.1, candidate paths for the node pair $(0,3)$ are path $0-1-3(42)$, path 0-2-3 (53), path 0-1-2-3 (413), and path 0-2-1-3 (51'2); candidate paths for pair(1,2) are path 1-2(1), path 1-3-2(23'), and path 1-0-2(4'5); candidate paths for pair(2,1) are path 2-1(1'), path 2-3-1(32'), and path 2-0-1(5'4). The number with dash indicates the nodes this path traverses, for example, $0-1-3$ stands for the path that starts from node 0 , traverses node 1 , and ends at node 3. The number in the parentheses after a path notation means all links this path traverse, e.g. the numbers in (42) after the path $0-1-3$ means that this path traverses link 4 and link 2. By enumerating candidate paths for all pairs, the set of all candidate paths for them forms Candidate Path Set (CPS) as the following:

Table 3.1: Candidate path set for four nodes network topology example

\begin{tabular}{|l|l|l|l|l|l|}
\hline Pair(0,1) & Pair (0,2) & Pair(0,3) & Pair(1,2) & Pair(1,3) & Pair(2,3) \\
\hline$(4)$ & $(5)$ & $(42)$ & $(1)$ & $(2)$ & $(3)$ \\
\hline$\left(51^{\prime}\right)$ & $(41)$ & $(53)$ & $\left(23^{\prime}\right)$ & $(13)$ & $\left(1^{\prime} 2\right)$ \\
\hline$\left(532^{\prime}\right)$ & $\left(423^{\prime}\right)$ & $(413)$ & $\left(4^{\prime} 5\right)$ & $\left(4^{\prime} 53\right)$ & $\left(5^{\prime} 42\right)$ \\
\hline & & $\left(51^{\prime} 2\right)$ & & & \\
\hline Pair (1,0) & Pair (2,0) & Pair(3,0) & Pair(2,1) & Pair(3,1) & Pair(3,2) \\
\hline$\left(4^{\prime}\right)$ & $\left(5^{\prime}\right)$ & $\left(2^{\prime} 4^{\prime}\right)$ & $\left(1^{\prime}\right)$ & $\left(2^{\prime}\right)$ & $\left(3^{\prime}\right)$ \\
\hline$\left(15^{\prime}\right)$ & $\left(1^{\prime} 4^{\prime}\right)$ & $\left(3^{\prime} 5^{\prime}\right)$ & $\left(32^{\prime}\right)$ & $\left(3^{\prime} 1^{\prime}\right)$ & $\left(2^{\prime} 1\right)$ \\
\hline$\left(23^{\prime} 5^{\prime}\right)$ & $\left(32^{\prime} 4^{\prime}\right)$ & $\left(3^{\prime} 1^{\prime} 4^{\prime}\right)$ & $\left(5^{\prime} 4\right)$ & $\left(3^{\prime} 5^{\prime} 4\right)$ & $\left(2^{\prime} 4^{\prime} 5\right)$ \\
\hline & & $\left(2^{\prime} 15^{\prime}\right)$ & & & \\
\hline
\end{tabular}


Selected Path Set (SPS): a SPS is a subset of CPS, which can satisfy the traffic demand matrix $T_{d}(\tau)$. Therefore it is a function of time period $\tau$. In the following, we will omit $\tau$ for simplicity.

With the given traffic demands $T_{d}\left(\tau_{1}\right)$ where $t_{0,3}\left(\tau_{1}\right)=450$ and $t_{2,1}\left(\tau_{1}\right)=350,\{(42)$, $\left.(53) ;\left(1^{\prime}\right),\left(32^{\prime}\right)\right\}$ is an SPS.

Norm of a SPS: the number of paths in a selected path set $S$ is its norm, denoted by $|S|$. With the given traffic demands $T_{d}$, there is a total of $N^{*}(N-1)$ pairs of nodes. For each pair of nodes $(x, y)$ which has nonzero traffic demands, there are certain number of paths, denoted by $|S|_{x, y}$, in SPS to accommodate the traffic demands related to node pair $(x, y)$. In the above example, there are two paths related to node pair $(0,3)$ and $(2,1)$, its norm is equal to four $\left(|S|=|S|_{0,3}+|S|_{2,1}=2+2=4\right)$.

Neighbor of a SPS,N(S): the set of all SPS with the same number of paths for each ingress-egress pair as SPS, $S$ is called the neighbor of $S$. Thus, $N(S)$ has the same norm $|S|$

Complete Solution Set, $C^{\text {ps }}$ : all possible solutions related to all SPS in accommodating given traffic demands are called the complete solution set $C^{s p s}$. $C^{s p s}$ is the whole solution space for the incoming traffic demands $T_{d}(\tau)$. 
Path vector, $P\left(r_{x y}^{i}\right):$ path vector contains several pieces of information: the identification of links in the path, the identification of the path (i) among node pair $x$ and $y$, the capacity of the path i.e. the minimum of the capacities of all links in the path, and the amount of traffic assigned to this path.

For example, $P\left(r_{x y}^{i}\right)=\left(l_{0}, l_{1}, l_{2}, \ldots, l_{M-1}, C_{x y}^{i}, S_{r_{x y}^{i}}\right)$ stands for a path vector related to path $r$ from node $x$ to node $y$. For each ingress-egress pair $x$ and $y$, there might be many paths. We will use the notation $i$ to identify the different paths related to ingress-egress pair $x$ and $y(i=1,2,3, \ldots) . \quad C_{x y}^{i}$ is defined as the capacity of the path which is the minimal capacity of all links in the path, and $S_{r_{x y}^{i}}$ is the traffic assigned to path $r_{x y}^{i}$. We will use bandwidth as units for both $C_{x y}^{i}$ and $S_{r_{x y}^{i}}$ in the following chapters. ${ }^{4}$ All nodes are sorted and each node is identified with an i.d. number from 0 to $N$-1. Supposing a link exists from node $i$ to node $j(0<i<j<N-1), l_{i}$ is defined as follows:

$$
l_{i}=\left\{\begin{array}{l}
1, \quad(i, j) \in r_{x y} \\
0, \quad(i, j) \notin r_{x y} \\
-1, \quad(j, i) \in r_{x y}
\end{array}\right.
$$

Below are the path vectors related to the example SPS $\left\{(42),(53) ;\left(1^{\prime}\right),\left(32^{\prime}\right)\right\}$

\footnotetext{
${ }^{4}$ Generally, we will use $r_{x y}$ to denote a path between ingress-egress nodes $x$ and $y$ when we do not need to
} identify all the paths among these two nodes. 
$P\left(r_{03}^{1}\right)=\left(0,1,0,1,0, C_{03}^{1}, S_{r_{03}^{1}}\right) \quad$ where $C_{03}^{1}=400$

$P\left(r_{03}^{2}\right)=\left(0,0,1,0,1, C_{03}^{2}, S_{r_{03}^{2}}\right)$ where $C_{03}^{2}=300$

$P\left(r_{21}^{1}\right)=\left(-1,0,0,0,0, C_{21}^{1}, S_{r_{21}^{1}}\right) \quad$ where $C_{21}^{1}=300$

$P\left(r_{21}^{2}\right)=\left(0,-1,1,0,0, C_{21}^{2}, S_{r_{21}^{2}}\right)$ where $C_{21}^{2}=300$

A SPS solution for this example can be $S_{r_{03}^{1}}=300, S_{r_{03}^{2}}=150, S_{r_{21}^{1}}=250, S_{r_{21}^{2}}=100$

Table 3.2: Link capacity table of the example network

\begin{tabular}{|l|l|l|}
\hline Links & Capacity of links & Available capacity after previous iteration \\
\hline$(1,2) / 1$ & 300 & 300 \\
\hline$(2,1) / 1 '$ & 300 & 50 \\
\hline$(1,3) / 2$ & 400 & 100 \\
\hline$(3,1) / 2$ & 400 & 300 \\
\hline$(2,3) / 3$ & 300 & 50 \\
\hline$(3,2) / 3$ & 300 & 300 \\
\hline$(0,1) / 4$ & 400 & 100 \\
\hline$(1,0) / 4$ & 400 & 400 \\
\hline$(0,2) / 5$ & 300 & 150 \\
\hline$(2,0) / 5$ & 300 & 300 \\
\hline
\end{tabular}

Link Capacity Table: This table contains the identity information of each link in the network. It also contains the nodes incident to each link, and the original capacity of each link. Moreover, the dynamically updated residual capacity for each link is also included. Table 3.2 is an example of a link capacity table after assigning traffic to links in the SPS: 
SPS generator function: The SPS generator function is used for generating a SPS. The input includes traffic demand matrix, traffic incident matrix, path vector, link capacity table, and a subset of a related CPS to accommodate the traffic demand matrix. The SPS generator function verifies if the subset is a selected path set. The output includes the SPS and its norm when the given subset is SPS; otherwise it provides an additional suggested path set. A detailed explanation about SPS generator function is represented in Fig. 3.2

The SPS generator function works in the following way. We can use the same network example and traffic demands $T_{d}\left(\tau_{1}\right)$ as above. We give an initial set of paths related to traffic demands $t_{0,3}\left(\tau_{1}\right)=450 ; t_{2,1}\left(\tau_{1}\right)=350 ;$ we choose this set from the CPS. Assume the set we have first chosen is $\left\{(42),(53) ;\left(1^{\prime}\right)\right\}$, the suggested path set is Null initially.

First we get path vector related to each pair. For path (42) and path (53) related to the pair $(0,3)$, we have a path vector as the following.

$P\left(r_{03}^{1}\right)=\left(0,1,0,1,0, C_{03}^{1}, S_{r_{03}^{1}}\right) \quad$ where $C_{03}^{1}=400$

$P\left(r_{03}^{2}\right)=\left(0,0,1,0,1, C_{03}^{2}, S_{r_{03}^{2}}\right)$ where $C_{03}^{2}=300$

Where $S_{r_{03}^{1}}=300, S_{r_{03}^{1}}=150$.

Notice that the total traffic assigned to path (42) and (53) is equal to $t_{0,3}\left(\tau_{1}\right)=450$; For path (1') related to pair $(2,1)$ 
$P\left(r_{21}^{1}\right)=\left(-1,0,0,0,0, C_{21}^{1}, S_{r_{21}^{1}}\right) \quad$ where $C_{21}^{1}=300$

The traffic demand $t_{2,1}\left(\tau_{1}\right)=350$ is more than the capacity of the path (1'), the given path set related to the pair $(2,1)$ could not accommodate the traffic demands. We can choose another path related to the pair $(2,1)$ from CPS, for example, path (32') and we add this path to the suggested path set. If we had assigned $S_{r_{21}^{1}}=250$ to path (1'), we could assign the path (32') in the suggested path set with assigned traffic of $S_{r_{21}^{2}}=100$; we then have path vector in the following:

$$
P\left(r_{21}^{2}\right)=\left(0,-1,1,0,0, C_{21}^{2}, S_{r_{21}^{2}}\right) \text { where } C_{21}^{2}=300, S_{r_{21}^{2}}=100
$$

Whereas the total assigned traffic is equal to the traffic demand $t_{2,1}\left(\tau_{1}\right)=350$.

The sum of the suggested path set $\left\{\left(32^{\prime}\right)\right\}$ and set of paths $\left\{(42),(53) ;\left(1^{\prime}\right)\right\}$ formed a solution of a SPS, i.e. $\left\{(42),(53) ;\left(1^{\prime}\right)\left(32^{\prime}\right)\right\}$. 


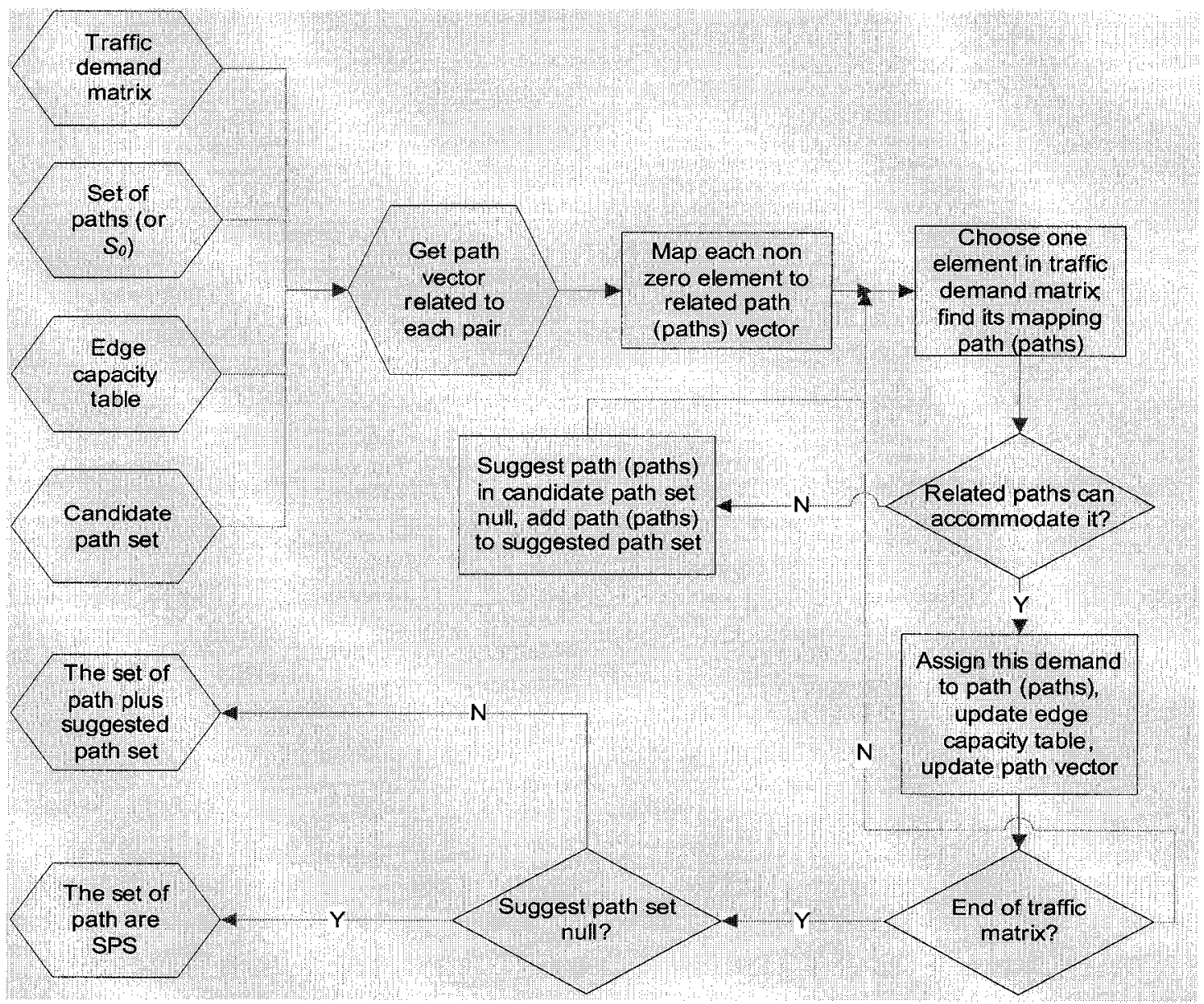

Fig. 3.2 SPS generator function flowchart 


\subsection{Formulation}

Our objective is to achieve minimum overall resource consumption and balance the load simultaneously. We define the distance of a path to be the sum of the inverses of the available bandwidths on all links along the path. This definition tries to strike a balance between link hop counts and available bandwidth. We will switch back to use the link notation defined earlier in this chapter. Supposing $U_{i j}$ is the unused bandwidth of link $(i$, $j$ ) where $(i, j)$ is a link in path $r_{x y}$. If we use $S_{i j}$ to denote the total traffic amount assigned to link $(i, j)$, then $U_{i j}=C_{i j}-S_{i j}$

It is easy to see

$S_{i j}=\sum_{(i, j) \in r_{x y}} S_{r_{x y}} \quad$ where $x \neq y ; x, y \in\{V\}$

Formula 3.3 shows the traffic assigned to link $(i, j)$ which equals the sum of the entire traffic assigned to each path in SPS (defined earlier) which traverses link $(i, j) . \quad V$ stands for the node set. The distance of a path $r_{x y}$ is therefore defined as

$$
D\left(r_{x y}\right)=\sum_{(i, j) \in r_{x y}} 1 / U_{i j}
$$


Given a topology $G(V, E)$ with $N$ nodes and related capacities for links, there is a total of $N^{*}(N-1)$ pairs of nodes. With given traffic demand, the objective function is formulated as follows:

$$
\underset{S \in C^{s p s}}{\operatorname{Min}} \sum_{r_{x y} \in S} D\left(r_{x y}\right) \quad \text { where } x \neq y ; x, y \in\{V\}
$$

Subject To:

1. $S_{i j} \leq C_{i j} \quad$ where $(i, j) \in r_{x y}$

2. $\sum_{r_{x y} \in S} S_{r_{x y}} \geq t_{x y} \quad$ where $x \neq y ; x, y \in\{V\}$

Where $r_{x y}$ stands for the paths between nodes $x$ and $y ; t_{x, y}(\tau)$ is the traffic demands between nodes $x$ and $y .\left(t_{x, y}(\tau)\right.$ is an element in traffic demand matrix $\left.T_{d}(\tau)\right) S_{i j}$ and $S_{r_{x y}}$ are the traffic assigned to link $(i, j)$ and path $r_{x y}$ respectively. While the total traffic assigned to link $(i, j), S_{i j}$, is equal to the sum of the traffic assigned to all paths $r_{x y}$ that traverse link $(i, j)$, as shown in the formula (3.3).

Here we give an example to show how the sum of distance over a SPS is calculated, using the $S P S$ example $\left\{(42),(53) ;\left(1^{\prime}\right),\left(32^{\prime}\right)\right\}$ in previous section. There are four paths in these $S P S, r_{03}^{1}, r_{03}^{2}, r_{21}^{1}$, and $r_{21}^{2}$. For path $r_{03}^{1}$, there are two links on this path, $(0,1)$ and $(1,3)$ (Fig. 3.1). The available bandwidths on link $(0,1)$ and $(1,3)$ are both $400-300=$ 100. Therefore, the distance for path $r_{03}^{1}$ is calculated as $1 / 100+1 / 100=0.02$. Similarly, 
the distances of path $r_{03}^{2}, r_{21}^{1}$, and $r_{21}^{2}$ can be calculated as $0.01333,0.02$, and 0.02333 respectively. Thus, the sum of the distances over this SPS example is 0.07666 .

Our objective function is to minimize the sum of distances of all selected paths between all ingress-egress pairs among complete solution set. The distance of each path is a combination of considering both load balance and network resource consumption, the sum of the distances of all selected paths between all ingress-egress node pairs is a combination of two objectives: load balance and resource consumption of the whole network.

Formula (3.4) indicates that each element in the distance formula is an indication of the utilization for the corresponding link. The presence of more available bandwidth results in decreased consumption of link utilization. When the available bandwidth of any link is close to zero, the distance of the related paths is close to infinity. The paths with large distances are eventually supposed to be discarded. In this way, highly congested paths in the multi-path routing scheme can be avoided. The summation is conducted over all the hops in each path. The distance of a path will increase with the increase in hop counts in the path. Thus, there is a choice of selecting either less-hop paths or lower-utilized paths. In our algorithm, we always choose the one with least distance.

To summarize, the paths in the optimized Selected Path Set should have as lower hop counts as possible, at the same time, we should aim to balance the load over all paths in order to keep the available bandwidth of each link as much as possible 
The calculation of formula (3.5) is time consuming because the available bandwidth along all links changes dynamically. Furthermore, the change of a path selection in one ingress and egress router pair might lead to changes among a few other pairs and finally make the calculation even more complex. To solve the problem within a reasonable time frame, the next chapter will deal with the simulated annealing technique used for developing an algorithm.

$-44-$ 


\section{Chapter 4}

\section{SDPSA Algorithm}

\subsection{Simulated annealing}

The SDPSA algorithm discussed in this thesis is based on the so-called annealing process. The term annealing is an analogy of an optimization technique to the cooling process of a liquid or solid. In an annealing process a metal is allowed to be cooled more slowly than usual and its interior structure will be more consistently; therefore, it will have much stronger crystal structures than their counterparts with faster cooling. Since Kirkpatrick et al. suggested simulated annealing technique in 1983 [41], it has been widely applied to optimization in areas such as the Very Large Scale Integration (VLSI) design, and the Traveling Salesman Problem (TSP) which is NP hard.

In the traditional local search algorithm, the final solution depends on the initial starting searching point. Various initial starting points usually lead to very different optimization results (see example in Fig. 4.1). In this figure, with the traditional local search algorithms, the starting point $\mathrm{P}$ will lead to the optimization result of point $\mathrm{Q}$, while the starting point $\mathrm{R}$ will lead to the final solution of either point $\mathrm{Q}$ or point $\mathrm{S}$. However, the simulated annealing method provides another optimization technique that is different 
from the traditional local search algorithms. In a simulated annealing process, the occasional uphill moves are conditionally accepted. Such accepted uphill moves allow skipping the local optimal point $Q$ or $S$ in the process of finding the global optimal point V.

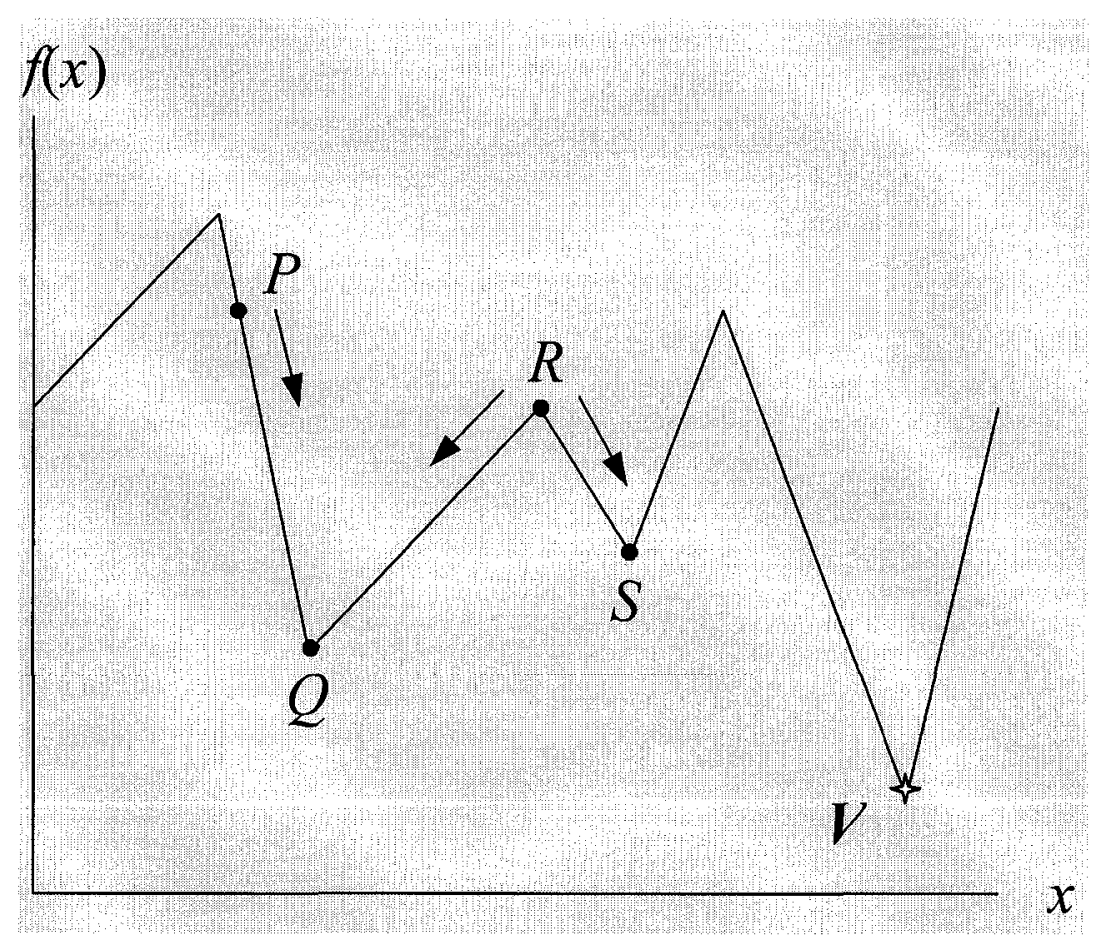

Fig. 4.1 Example of searching for optimal point

Fig. 4.2 provides a diagram of the simulated annealing process [42]. For each temperature the simulated annealing algorithm runs through a number of cycles. As the cycle runs the inputs are randomized. Only randomization that produces a more suitable set of inputs will be kept. Once the specified number of cycles has been completed, the temperature can be lowered. Once the temperature is lowered, it is determined if the temperature has reached the lowest allowed temperature. If the temperature is not lower than the lowest allowed temperature, then the temperature is lowered again and another $-46-$ 
cycle of randomizations will take place. If the temperature is lower than the minimum allowed temperature, the simulated annealing algorithm is completed.

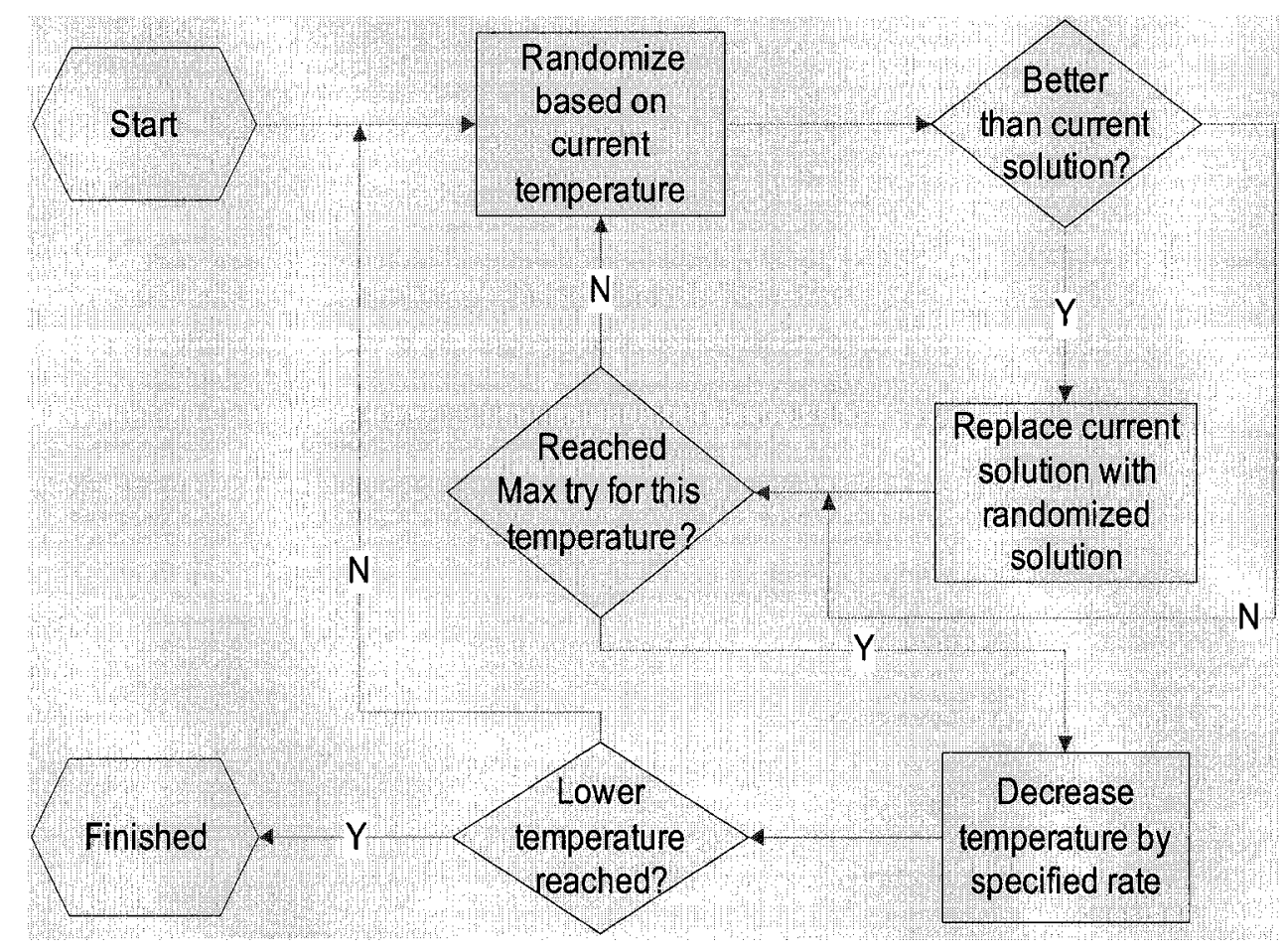

Fig. 4.2 Simulated annealing process

The simulated annealing scheme for finding the optimal solution can be applied to solving the multi-path routing optimization problem (see Chapter 3 ). In order to find a Selected Path Set satisfying our objective function in formula 3.5 , we need to take a further look at the distance formula 3.4. In 3.4, each element on the right-hand side is an indication of the utilization of each link. The summation part indicates the total hops in each path. In summary, the paths in the optimized selected path set should have as fewer hops as possible, and at the same time, balance the load over all paths to keep available bandwidth as much as possible on each link. 
In order to accommodate the traffic demands, the paths of some node pairs (or all node pairs) in the network (depending on the traffic demand matrix) will be selected and the traffic will be assigned to each path according to its link capacity. For each particular node pair in the network, when the number of paths increases in the Selected Path Set, the link utilization in each path will decrease. On the other hand, the link utilization will increase if fewer paths are selected for the traffic demand related to this particular node pair. Moreover, the total number of paths in a Selected Path Set, i.e., the norm of Selected Path Set, is an important parameter in the algorithm. When the norm of a Selected Path Set is less, there are more options of path selection for each node pair from the Candidate Path Set. This case is similar to the one that has higher temperature in the simulated annealing process. With the increase of path number in Selected Path Set, there are fewer options of path selection for the candidates of the Selected Path Set. This indicates that the path selection process for a Selected Path Set is more stable. This process is similar to the one that has lower temperature in the simulated annealing process. 


\subsection{SDPSA algorithm}

As we mentioned previously, the optimization problem discussed in this thesis is very similar to an annealing process, and thus simulated annealing optimization is applied. As discussed above, the traffic metrics do not change in a certain time period regardless of the duration of this period. In a given time period $\tau_{1}$, we assume that the sub-optimized solution is available with a given path set which can accommodate related traffic demands with minimized objective function. As the time moves to the next period, $\tau_{2}$, the traffic demand metrics change, and consequently, some or all of the paths related to individual ingress and egress pairs should be changed in order to reach new optimal solution. As shown in Fig. 4.4, our newly developed SDPSA algorithm does take advantage of the previous optimal solution to achieve the current objective.

As shown in both Fig. 4.3 and Fig. 4.4, there are two loops in the SDPSA algorithm. The inner loop searches for a better solution among those SPSs with the same norm. The SPS with the same norm is the analogy of the temperature in simulated annealing. The SPS (noted as $S$ ) with the same norm here stands for the number of paths that have been selected according to each ingress and egress pair $\left(|S|_{x, y}\right)$ are equal.

$|S|=\sum_{r_{x, y} \in S}|S|_{x, y} \quad$ Where $x \neq y ; x, y \in\{V\}$ 
All the SPSs with the same temperature also mean that the selection of all neighbors is based on the same selected path set $S_{0}$.

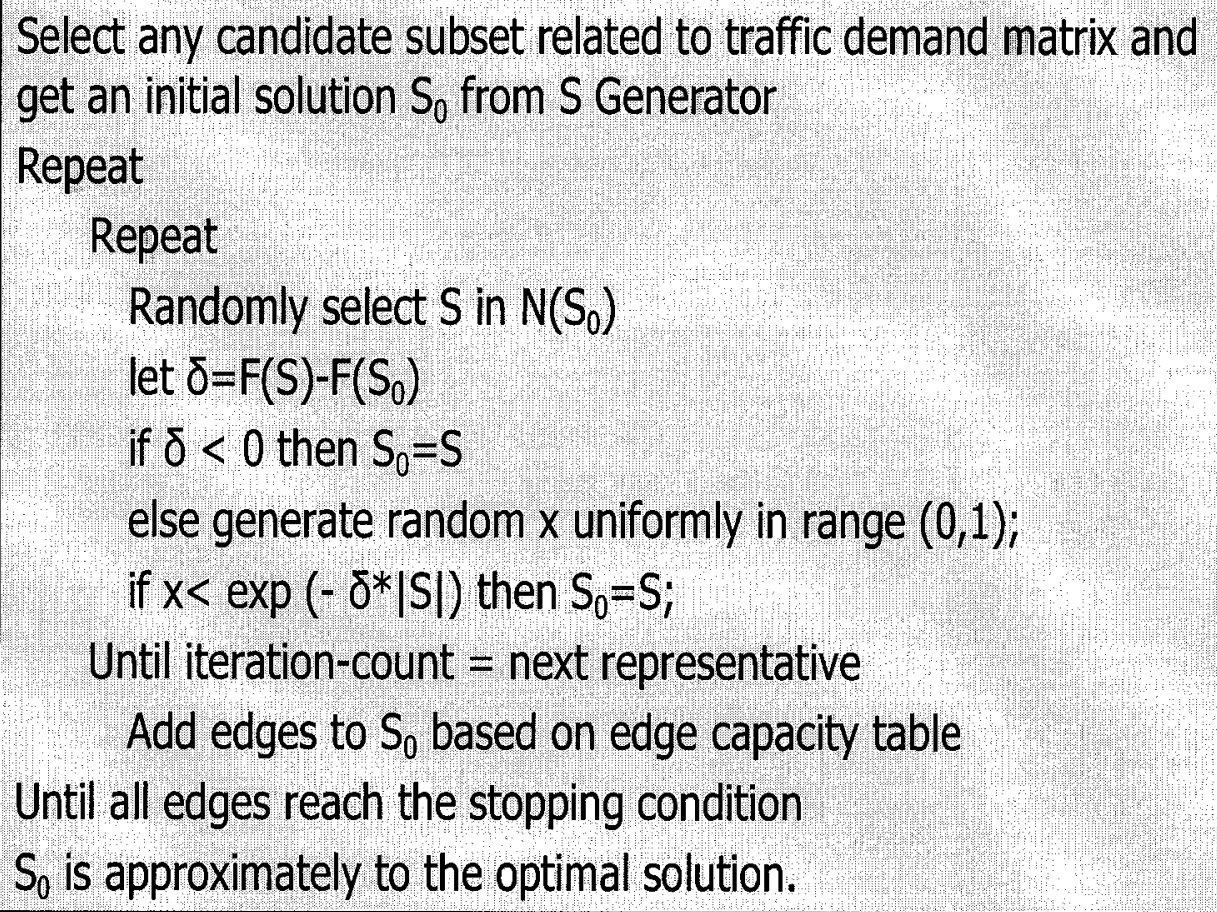

Fig. 4.3 SDPSA algorithm

In Fig. 4.4, parameter $\delta$ is defined as the difference between the currently selected sample of distance of $S$ and its initial solution's distance of $S_{0}$, where,

$$
\begin{gathered}
F(S)=\sum_{\substack{r_{x y} \in S \\
S \in N\left(S_{0}\right)}} D\left(r_{x y}\right) \\
F\left(S_{0}\right)=\sum_{r_{x y} \in S_{0}} D\left(r_{x y}\right)
\end{gathered}
$$


Here $S$ is also a selected path set. Whenever a better solution is found, i.e. $\delta<0$, the currently best solution will be updated; otherwise, a random number $x$ between $(0,1)$ is generated. If $x<\exp \left(-\delta^{*}\left|N-S_{0}\right|\right)$, the current best solution will still be updated. The iteration continues until the maximum number of repetitions is reached.

To give an illustration, we use the SPS example in chapter 3.2 as our initial start SPS, $S_{0}$. The sum of the distance over $S_{0}, \mathrm{~F}\left(S_{0}\right)$, is 0.07666 . We randomly select $\mathrm{S}$ from $\mathrm{N}\left(S_{0}\right)$, $\left\{(42),(53) ;\left(1^{\prime}\right),\left(5^{\prime} 4\right)\right\}$ and calculate the sum of the distance of this $S, F(S)$, which is equal to 0.1983 . $\delta$ is equal to 0.1216 which is bigger than zero. We generate a random number $x$ between $(0,1)$ and compare $x$ to $\exp \left(-0.1216^{*} 4\right)=0.615$. The current best solution will be updated if $\mathrm{x}$ is less than 0.0615 (The current best solution will be kept if $\mathrm{x}$ is bigger than 0.0615). The iteration continues until the maximum number of repetitions is reached.

The outer loop increases the number of paths similar to the decrease of temperature in the simulated annealing process until the stopping condition is met. Indeed, the solution of the last time period can be used as the initial solution of the current time period. As shown in Fig. 4.4, if previous solution, say $S_{0}$, can accommodate current traffic demands, the algorithm will simply take $S_{0}$ as the current solution.

As can be seen from the algorithm in Fig. 4.3, the SDPSA algorithm allows probabilistic acceptance of non-improving moves. The acceptance probability depends on the control 
parameter, $|S|$, and the amount of costs increases, $\delta$. The acceptance probability is high for a small $\delta$ and $|S|$. As $|S|$ increases, the acceptance probability will decrease accordingly.

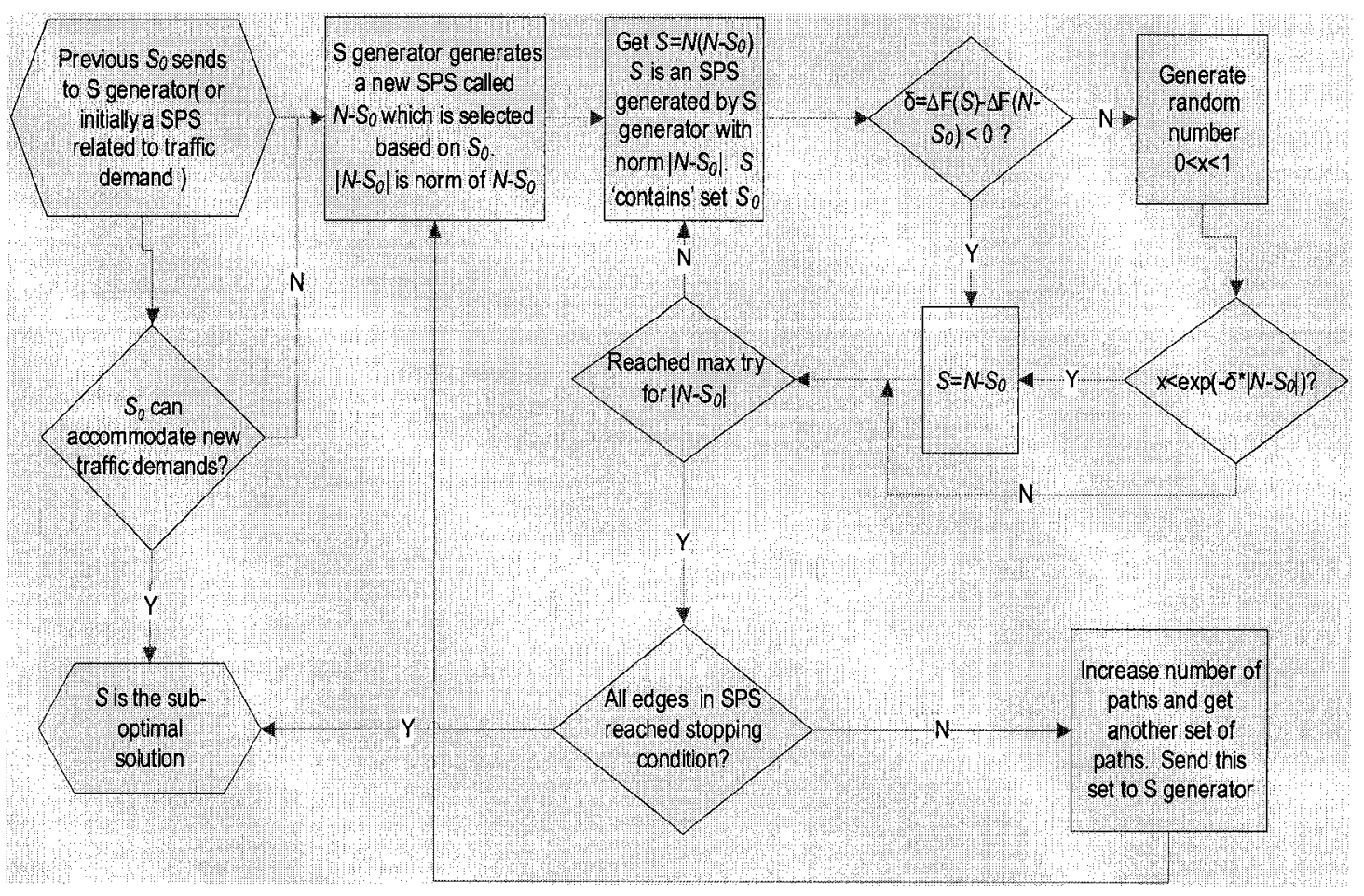

Fig. 4.4 SDPSA algorithm flowchart 


\subsection{Complexity analysis for the HSTwp algorithm and the SDPSA algorithm}

The HSTwp algorithm (see Chapter 2 and Fig. 2.3 ) contains three parts: preprocessing the given graph, finding multiple paths, and calculating load-splitting ratios. The complexity analysis of the HSTwp algorithm is as following: firstly, in the graph conversion problem, the computation complexity is bounded by $O(|M+N|)$ where $M$ is the number of links and $N$ is the number of nodes in the graph. Secondly, in the $K$ widest paths problem, the best known bound for the ordered set is $\mathrm{O}\left(N^{3}\right)$. Finally, the algorithm for splitting the traffic demand into $K$ paths is bound by $O(|K \log K|)$, and calculating the load splitting ratio for each path is bound by $O(|2 K|)$ where $K$ is the number of paths selected. Therefore, the time complexity for the HSTwp algorithm is bound by $O(M+$ $\left.N^{3}+|K \log K|\right)$. The above complexity analysis is adopted from [22].

The SDPSA algorithm also consists of three parts: preparing the candidate path set, calculating the initial selected path set depending on traffic demand of the first time period, and performing simulated annealing process. Firstly, in preparing the candidate path set for each node pair, the computation complexity is $O(N !)$ for complete $\operatorname{graph}^{5}$. The average node degree for a complete graph is $N-1$ while the average node degree for common network topology is between 2.5 to 5 [43]. Finding $K$ paths between each pair of nodes in the network to be the candidate path set is the alternative solution for finding

\footnotetext{
${ }^{5}$ A graph is defined as a complete graph if each node has links connected with all other nodes in the graph.
} $-53-$ 
the candidate path set. The complexity of finding $K$ paths for a pair of nodes is bounded by $O\left(N^{3}\right)$ [44]. There is a total of $N^{*}(N-1)$ pairs where $N$ is the number of nodes. Therefore the total computation complexity for this computing $K$ paths for each pair of nodes in the network as our candidate path set is $O\left(N^{5}\right)$. However, we only need to calculate this part offline for one time only unless the topology changes. Second, in the initial selected path set, the worst case for calculating a selected path set, with $S$ generator function, is $O\left(N^{2}{ }^{*} K M \log N\right)$ where $M$ is the number of links, $N$ is the number of nodes, and $K$ is the number of paths for any pair of nodes in a given topology. Each path has at most N-1 links, sorting the available bandwidth among all links in a path to find the bottleneck link is bounded by $\mathrm{O}(\log \mathrm{N})$. After reserving the bandwidth for each path, updating the link capacity table is bounded by $\mathrm{M}$ and there are at most $\mathrm{K}$ paths for each pair of nodes. Here we assume the traffic in the network is not overloaded such that we can find paths among each pair of node to be a SPS. In the worst case, there are traffic demands for all pairs of nodes in the network. Calculating a selected path set and assigning traffic to the paths in the SPS is bounded by $O\left(N^{2} * K M \log N\right)$. Finally, for the simulated annealing process, we simply use previous SPS if the previous solution can accommodate the current future traffic. Otherwise, SPS will be re-calculated and the time complexity is bounded by $O\left(N^{2} * K M \log N\right)$. The total number of paths in candidate path set is $K^{*} N^{*}(N-1)$. There are various numbers for norm of SPS, $|S|$ where the norm is ranging from 1 to $K^{*} N^{*}(N-1)$ (For the simplest case, a SPS with one path accommodate related traffic demand; for the worst case, all $K^{*} N^{*}(N-1)$ paths in candidate path set are selected as SPS to accommodate the traffic demand). If, we increase the norm by one each time, the time complexity for the simulated annealing process is bound by 
$O\left(N^{4} * K^{2} M \log N\right)$. In a more coarse way, we can increase the norm of $S P S$ by at most $N^{2}$ links each time until number of paths related to each pair reaches the limit $K$. The complexity for the simulated annealing process is then reduced to $O\left(N^{2} * K^{2} M \log N\right)$ as in Table 4.1

Table 4.1: Complexity analysis for HSTwp algorithm and SDPSA algorithm

\begin{tabular}{|c|c|c|c|c|c|c|}
\hline Algorithm & \multicolumn{3}{|c|}{ HSTwp algorithm } & \multicolumn{3}{|c|}{ SDPSA algorithm } \\
\hline Step 1 & $\begin{array}{c}\text { graph } \\
\text { conversion } \\
\text { problem }\end{array}$ & $O(|M+N|)$ & 总 & $\begin{array}{l}\text { preparing } \\
\text { the } \\
\text { candidate } \\
\text { path set }\end{array}$ & $O\left(N^{5}\right)$ & 우우 \\
\hline Step 2 & $\begin{array}{c}K \text { widest } \\
\text { paths } \\
\text { problem }\end{array}$ & $\mathrm{O}\left(N^{3}\right)$ & 委 & $\begin{array}{c}\text { calculating } \\
\text { a selected } \\
\text { path set }\end{array}$ & $O\left(N^{2} * K M \log N\right)$ & ald \\
\hline Step 3 & $\begin{array}{l}\text { algorithm } \\
\text { for splitting } \\
\text { the traffic } \\
\text { demand } \\
\text { into } K \text { paths }\end{array}$ & $O(|K \log K|)$ & 象 & $\begin{array}{l}\text { simulated } \\
\text { annealing } \\
\text { process }\end{array}$ & $O\left(N^{2} * K^{2} M \log N\right)$ & 미 \\
\hline Total & \multicolumn{3}{|c|}{$O\left(M+N^{3}+|K \log K|\right)$} & \multicolumn{3}{|c|}{$O\left(N^{2} * K^{2} M \log N+N^{5}\right)$} \\
\hline
\end{tabular}

Legend: Need recalculate each time when traffic demand changes.

이: Might need recalculate when traffic demands change.

우: Need to calculate for one time only when topology is given initially. 
Based on the complexity analysis above, the SDPSA algorithm is more complex than the HSTwp algorithm. We will have a discussion for this next. Firstly, the candidate path set is calculated off-line and then it can be used unless some link fails or topology changes. Secondly, the worst case for calculating a selected path set may not occur very often because the simulated annealing process takes advantage of the previous period multi-path routing information as an initial point of the current period. Thirdly, we can set different stopping conditions in the simulated annealing process to reduce the complexity like in Table 4.1. Finally, the number of nodes and links in a backbone network is not large enough (typically less than 30 nodes) to cause the scalability problem. 


\section{Chapter 5}

\section{Performance Analysis}

In this chapter, the performances of SDPSA algorithm are compared with HSTwp algorithm in two scenarios: a random generated network topology (scenario I), and a real network example, NSF network (scenario II).

\subsection{Simulation setup for scenario I}

The performance of the SDPSA algorithm is investigated by applying the algorithm to a randomly generated topology example. The topology shown in Fig. 5.1 is generated by the BRITE topology generator [43], which can generate flat topologies. It is assumed that the links are symmetric with the same capacity in both directions (Fig. 5.1); the model parameters for generating this topology are Alpha $=0.15$ and Beta $=0.20$, where Alpha indicates the relationship between short and long physical distance of all links, and Beta measures the degree of connectivity in the network. The topology example has six nodes and ten bi-directional links. Link capacities are obtained from the BRITE package by using the uniform distribution over the range of $\left\{\begin{array}{lll}300 & 1000\end{array}\right\}$ megabyte bandwidth units. The traffic demands are asymmetric as in the common cases in [22]. The traffic demands are generated from randomization of an initial static traffic demand matrix of 
$\left\{t_{05}=600, t_{14}=500, t_{2 I}=400, t_{54}=300\right\}$ and we use megabyte bandwidth units as the traffic demands.

The simulation for both scenario I and scenario II were performed on PC running on Windows 2000 operation system. The simulation code was implemented in $\mathrm{C}$ and Matlab.

Fig. 5.3 shows the traffic demands among four pairs of nodes in various time periods. In order to examine the algorithm sensitivity to variations of traffic demands, these demands in each time period are randomly selected from the set of the random variations with the different percentage of the initial static traffic demand matrix. The percentages are taken from $\{10 \%, 20 \%, 30 \%, 40 \%, 50 \%, 60 \%, 70 \%, 80 \%, 90 \%$, and $100 \%\}$; the traffic demands for various time periods are dynamically changing.

By applying a well-known heuristic HSTwp algorithm reported in [22] and the proposed SDPSA algorithm to the scenario above, two groups of different multi-path routing solutions are generated through various time periods. Each solution includes related route information such as the number of paths and hops used, the interference links appeared, and the total resources consumed. We will analyze the simulation result in the next section to show that the SDPSA algorithm has a superior performance over the HSTwp algorithm in terms of overall network resource consumption. 


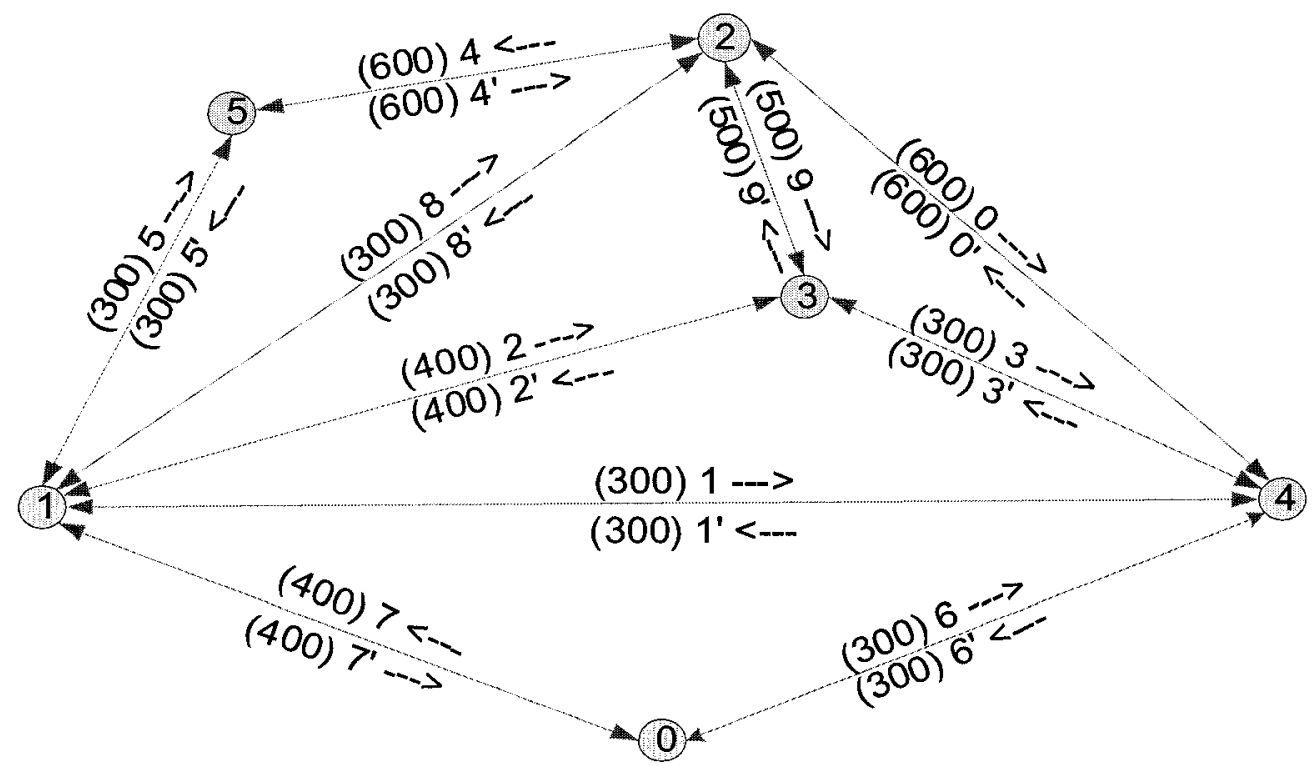

Fig. 5.1 Simulation Topology with 6 nodes and 10 bi-directional links

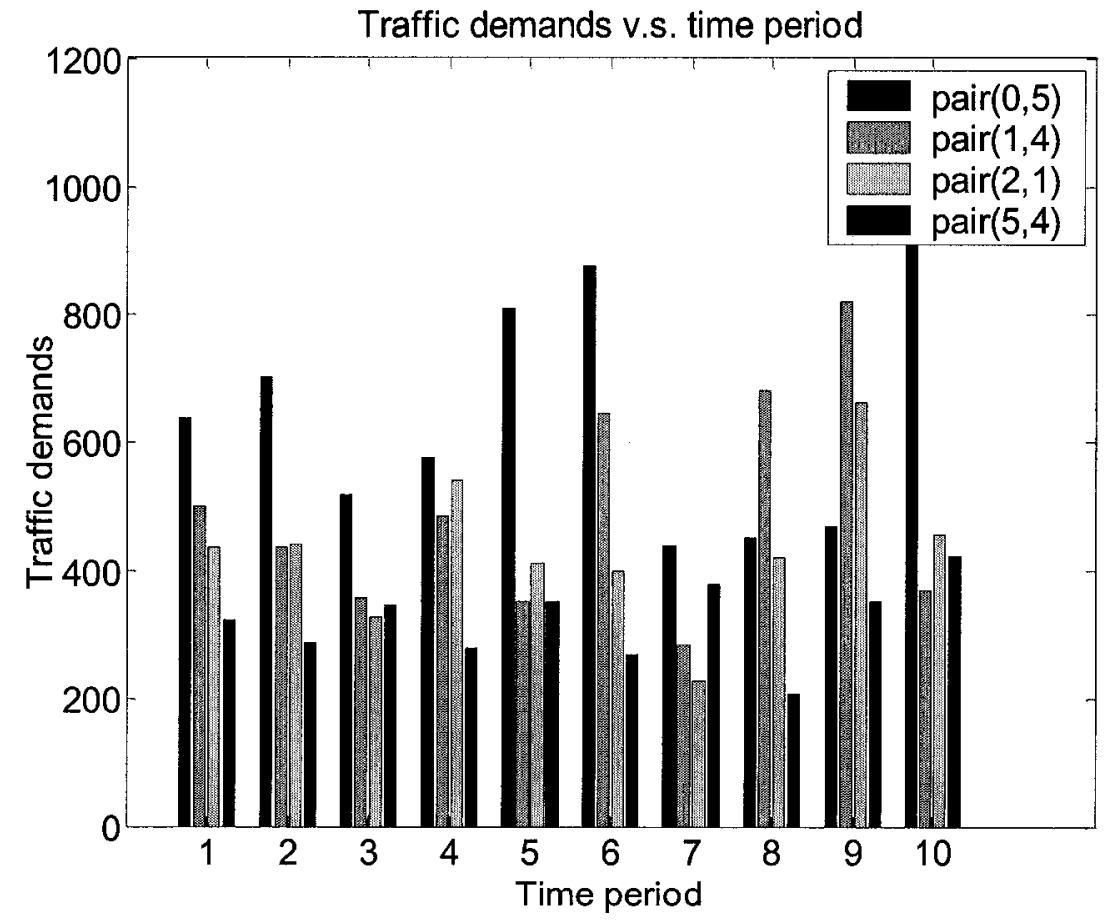

Fig. 5.2 Traffic demands in each time period. 


\subsection{Simulation results for scenario I}

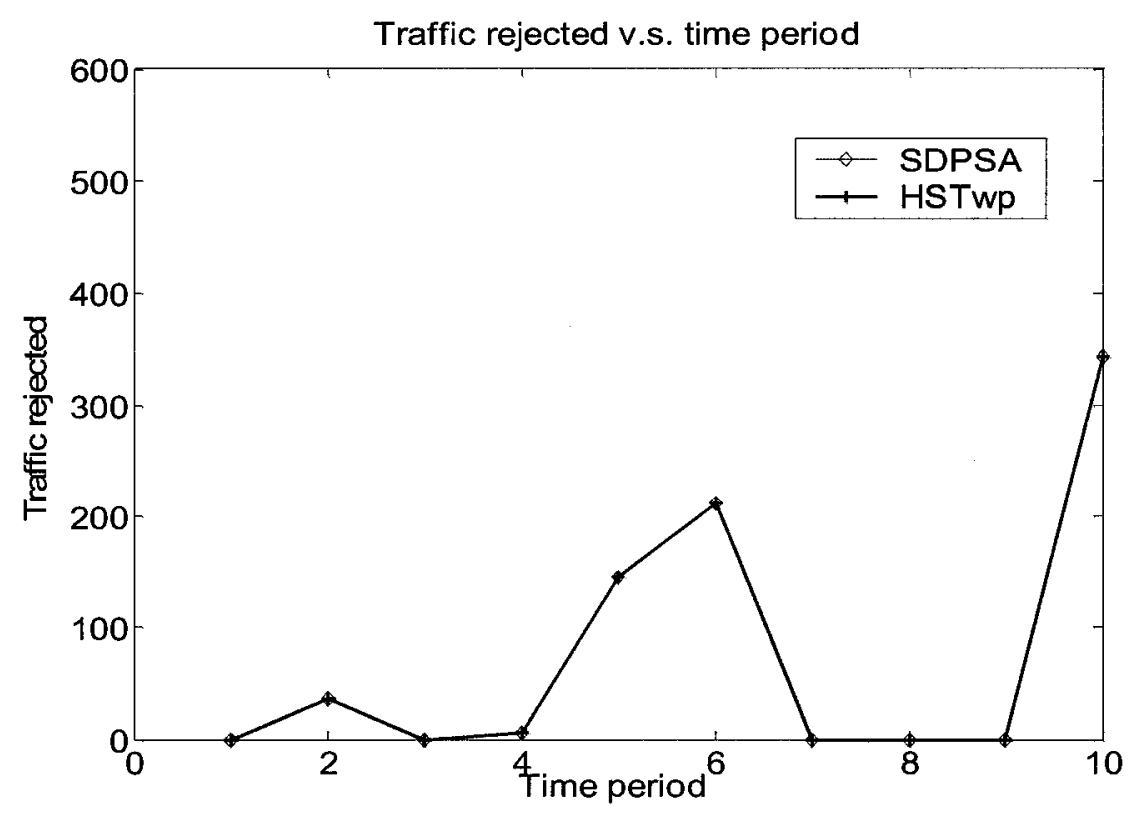

Fig. 5.3 Rejected traffic v.s time period

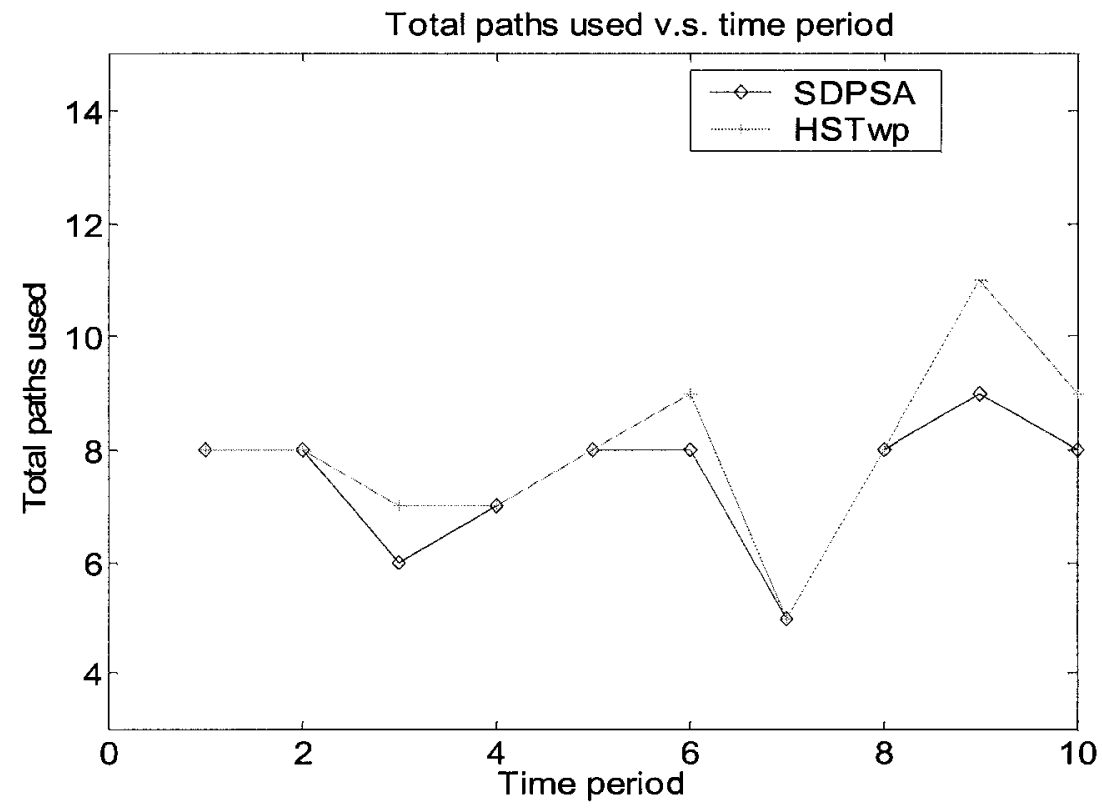

Fig. 5.4 Total paths used v.s. time period

$-60-$ 


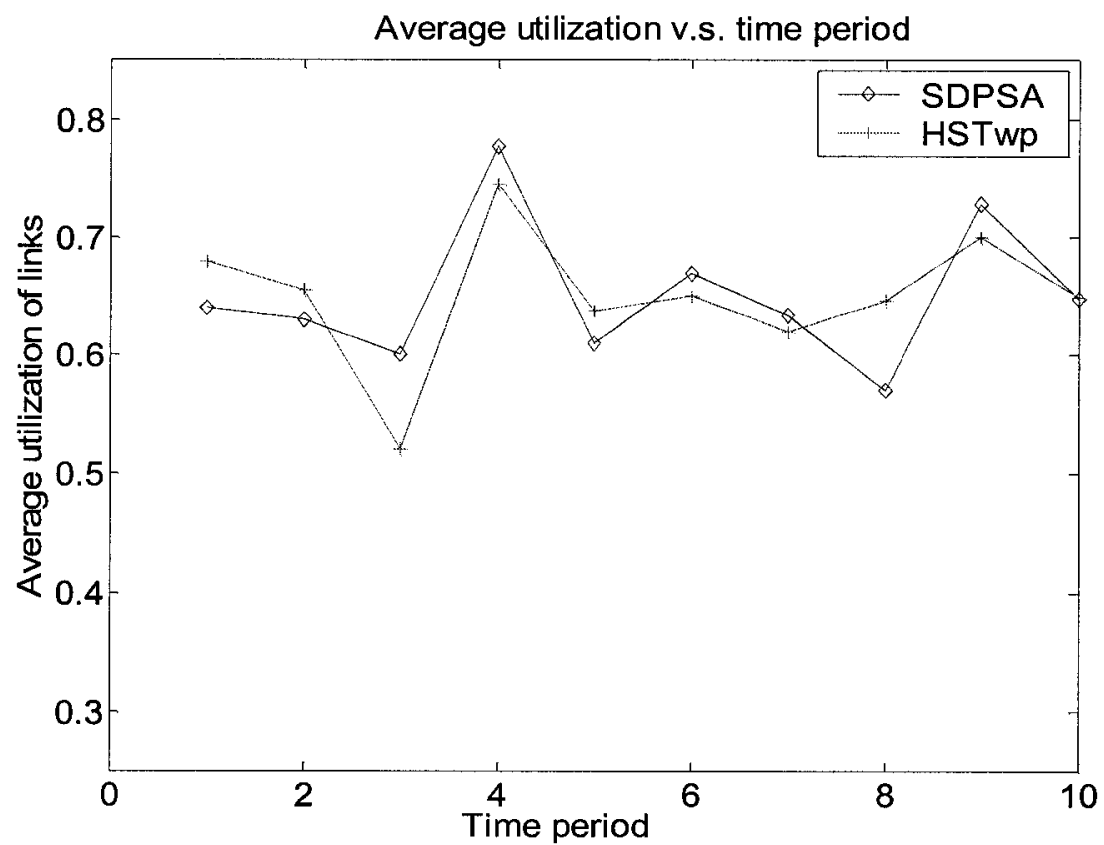

Fig. 5.5 Average utilization among all selected links

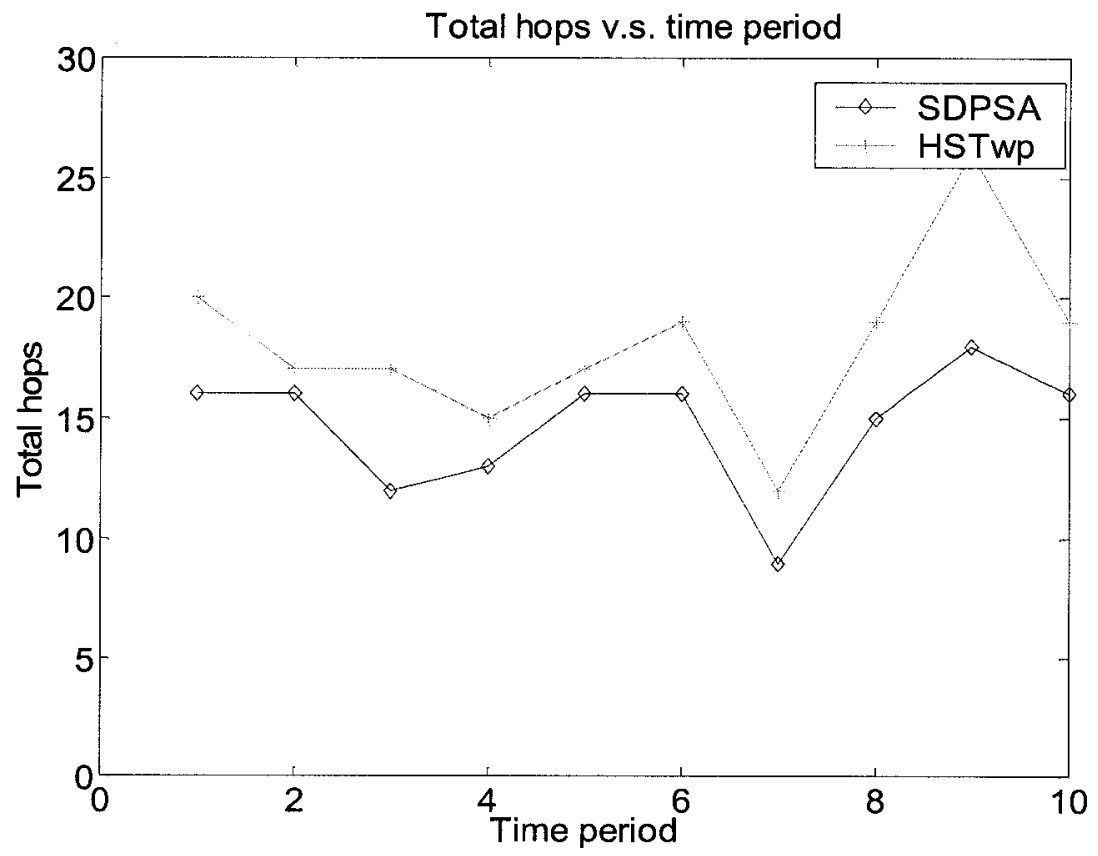

Fig. 5.6 Total hops among all selected paths.

$-61-$ 


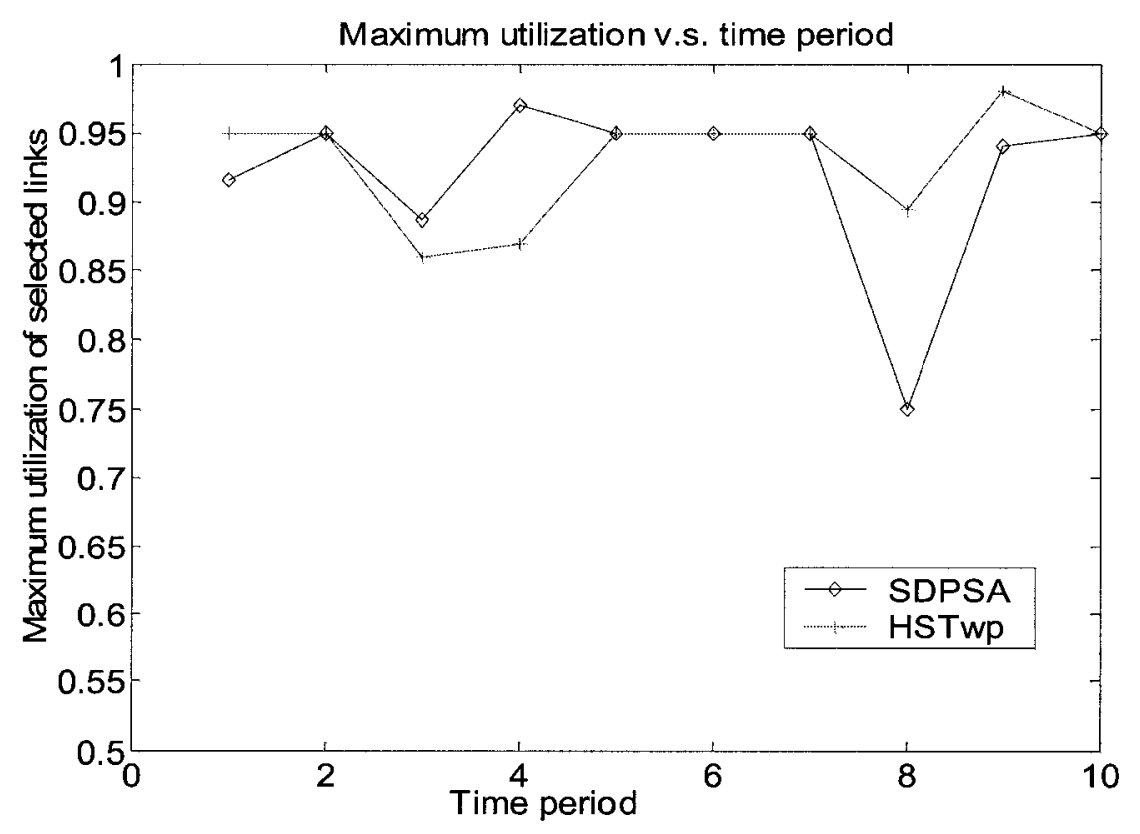

Fig. 5.7 Maximum utilization among all selected links

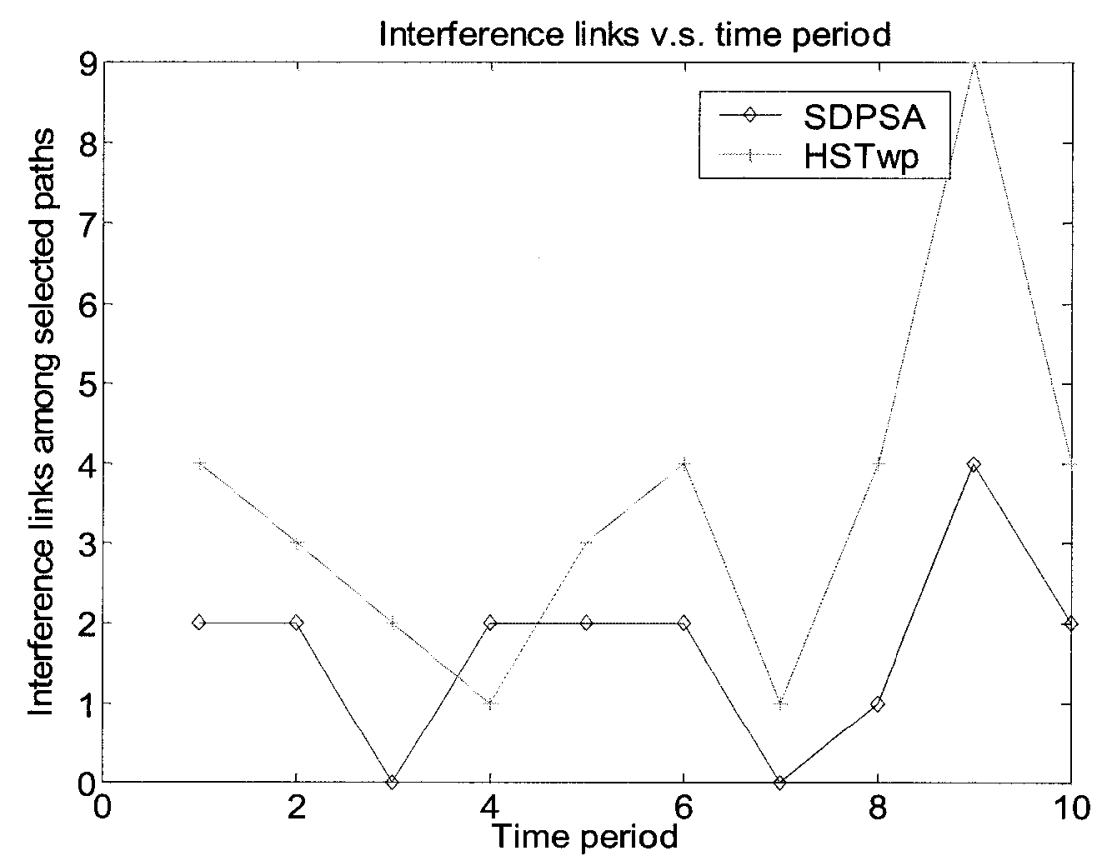

Fig. 5.8 Total interference links among all selected paths

$-62-$ 
Fig. 5.2 shows that the volumes of rejected traffic in various time periods for the SDPSA algorithm and the HSTwp algorithm are identical. Thus, with the same traffic demands in various time periods in Fig. 5.3, the SDPSA algorithm and the HSTwp algorithm can find their own multi-path routing solutions to accommodate the same amount of traffic demands in different time periods. In Fig. 5.4, Fig. 5.6, and Fig. 5.8, various parameters such as paths, hops, and interference links related to network resources consumption are compared for these two algorithms throughout various time periods. The number of hops used in the SDPSA algorithm is consistently less than that of the HSTwp algorithm in Fig. 5.6, whereas the amount of paths used in the SDPSA algorithm is no more than that of the HSTwp algorithm in Fig. 5.4. In Fig. 5.8, the link interference appeared in the SDPSA algorithm is no more than that of the HSTwp algorithm except for time period four. This indicates that the SDPSA algorithm consumes less network resources in admitting the same amount of traffic demands. This result can also be seen in Table 5.1 below.

In Fig. 5.5 and Fig. 5.7, average utilization and maximum utilization for all selected links are compared throughout various time periods for the SDPSA algorithm and the HSTwp algorithm. Based on the results, it is difficult to conclude which of the two algorithms consumes less utilization in general. However, it is worthy to note that the interference links which occurred in each algorithm might affect the average and maximum utilization of related algorithms. For instance, the interference links in the SDPSA algorithm is higher than that of the HSTwp algorithm in time period four, both the related average and 
maximum utilization for the SDPSA algorithm exceeded corresponding levels in the HSTwp algorithm in Fig. 5.5 and Fig. 5.7.

Table 5.1 lists the total network resource consumption using the HSTwp and SDPSA algorithms. The total network resource consumption is calculated by adding the product of the bandwidth assigned to each of the selected paths $S_{r_{x y}}$ and the hop count $r_{x y}^{\text {hops in }}$ each path.

$$
R=\sum_{r_{x y} \in S}\left(S_{r_{x y}} \times r_{x y}^{h o p s}\right) \quad \text { Where } x \neq y ; x, y \in\{V\}
$$

According to the formula $S_{i j}=\sum_{(i, j) \in r_{x y}} S_{r_{x y}}$ in Chapter 3, the total network resource consumption can also be expressed as:

$$
R=\sum_{\substack{(i, j) \in r_{x y} \in S \\ x, y \in\{V\} ; x \neq y}} S_{i j}
$$

From Table 5.1, it can be seen that on average the SDPSA algorithm consumes $12.9 \%$ less network resources than the HSTwp algorithm does. The maximum resource saving can reach up to $26.6 \%$, which is more than twice of the average resource saving. This indicates that the SDPA algorithm can successfully meet our objective of reducing total 
network resource consumption. As a result, by using the SDPSA algorithm, the network can accommodate more traffic demands.

Table 5.1: Total resources consumed on all selected path

\begin{tabular}{|c|c|c|c|}
\hline Time & $\begin{array}{c}\text { Resources used } \\
\text { in SDPSA }\end{array}$ & $\begin{array}{c}\text { Resources used } \\
\text { in HSTwp } \\
\left(R_{\text {SDPSA }}\right)\end{array}$ & $\begin{array}{c}\text { Resources gain } \\
\left(\left(R_{\text {HSTwp }}\right.\right.\end{array}$ \\
\hline 1 & 3841 & 4532 & 0.180 \\
\hline 2 & 3500 & 3911 & 0.117 \\
\hline 3 & 3206 & 3542 & 0.105 \\
\hline 4 & 3560 & 3911 & 0.099 \\
\hline 5 & 3570 & 3870 & 0.084 \\
\hline 6 & 3989 & 4215 & 0.057 \\
\hline 7 & 2440 & 3061 & 0.255 \\
\hline 8 & 3355 & 4247 & 0.266 \\
\hline 9 & 4394 & 5081 & 0.156 \\
\hline 10 & 3948 & 4077 & 0.033 \\
\hline Average & 3580.3 & 4044.7 & 0.129 \\
\hline
\end{tabular}

$-65-$ 


\subsection{Simulation setup for scenario II}

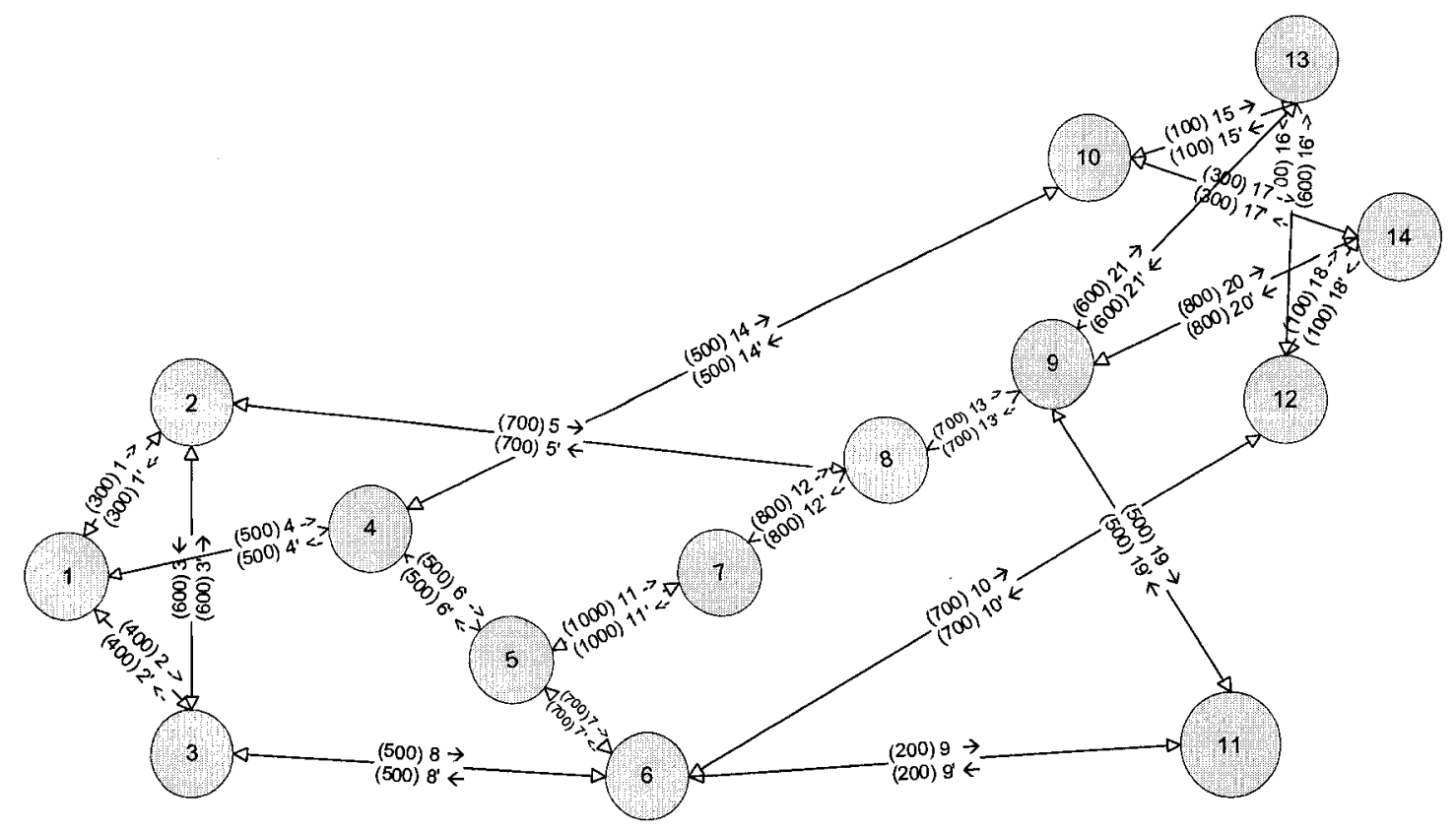

Fig. 5.9 NSFNET Topology with 14 nodes and 21 bi-directional links

In this section and the section follows, the network performance of SDPSA algorithm will be investigated using the NSFNT topology with 14 nodes and 21 bi-directional links as shown in Fig. 5.9.

Different from scenario I which is a randomly generated simpler network topology, the NSFNET is a well known practical core network example. The NSFNET is a high speed "network of networks" which is hierarchical in nature. At the highest level is a network that spans the continental United States. NSFNET stands for the National Science Foundation's network, which started as a series of networks devoted to research and education communication, funded by the U.S. government, and it superseded ARPANET as the backbone of the Internet. It has since been replaced by commercial networks. 
The performance of our SDPSA algorithm is analyzed using this real backbone network topology. Again, the HSTwp algorithm is performed on the same topology for comparison.

In Fig. 5.9, each bi-directional link has two identification numbers marking its two directions. The capacities of the bi-directional links are symmetric, and they are shown inside the parentheses. Similar to scenario I, the link capacities are obtained by using a uniform distribution over the range of $\left\{\begin{array}{ll}300 & 1000\end{array}\right\}$ Megabytes bandwidth unit. The traffic demands are asymmetric as that is the common case in [22]. The traffic demands are generated from randomization of an initial static traffic demand matrix of $\left\{t_{l, 8}=400\right.$, $\left.t_{2,6}=700, t_{11,13}=500\right\}$ and we use megabyte bandwidth units as the traffic demands.

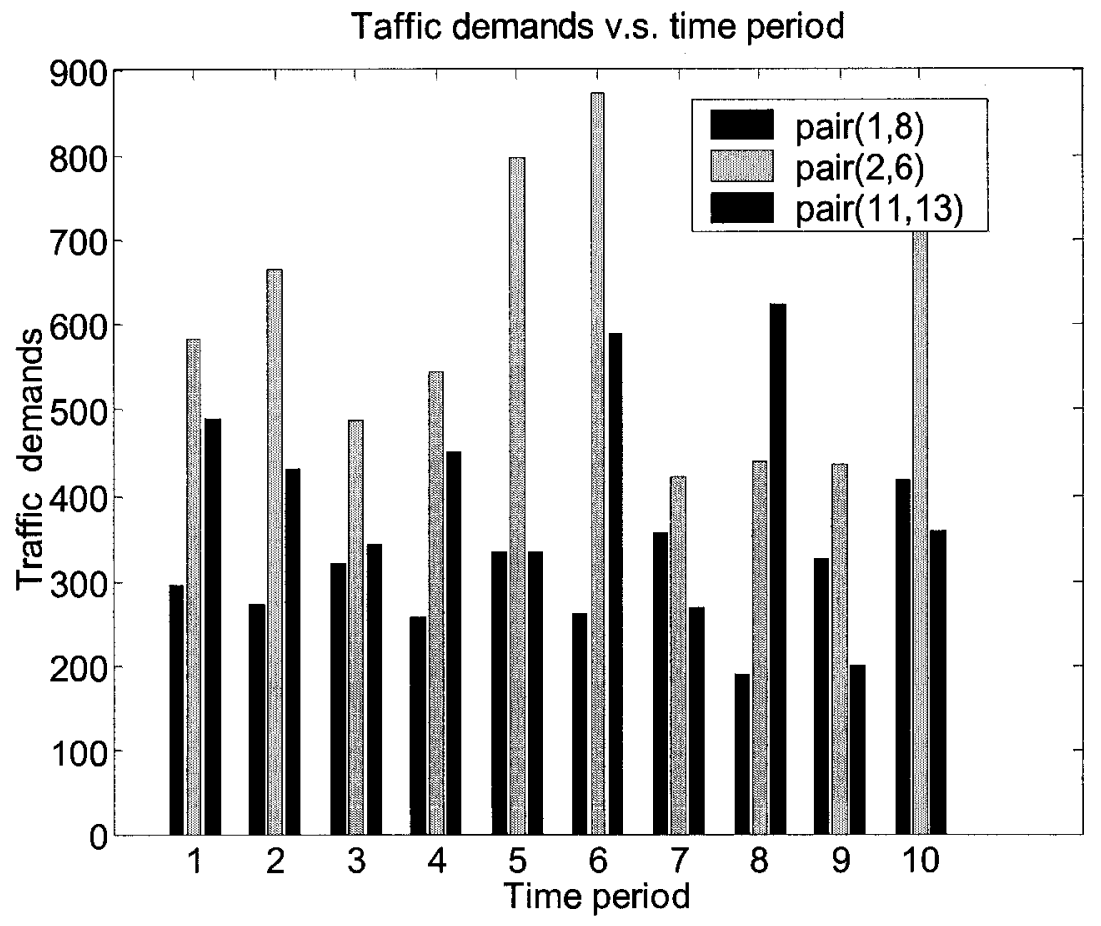

Fig. 5.10 Traffic demands in each time period for NSFNET

$-67-$ 
Fig. 5.10 shows the traffic demands among three pairs of nodes in various time periods. In order to examine the algorithm sensitivity to variations of traffic demands, like in scenario I, the demands in each time period are randomly selected from the set of the random variations with the different percentage of the initial static traffic demand matrix. The percentages are taken from $\{10 \%, 20 \%, 30 \%, 40 \%, 50 \%, 60 \%, 70 \%, 80 \%, 90 \%$, and $100 \%$ s similar to scenario I.

In scenario II, the heuristic HSTwp algorithm and the SDPSA algorithm are applied on the NSFNET topology, and different multi-path routing solutions related to each algorithm are generated through various time periods. Each solution includes related route information such as the number of paths and hops used, the interference links appeared, and the total resources consumed. The simulation result is analyzed in the next section to show that the SDPSA algorithm has a superior performance over the HSTwp algorithm in terms of overall network resource consumption in the NSFNET topology. 


\subsection{Simulation results for scenario II}

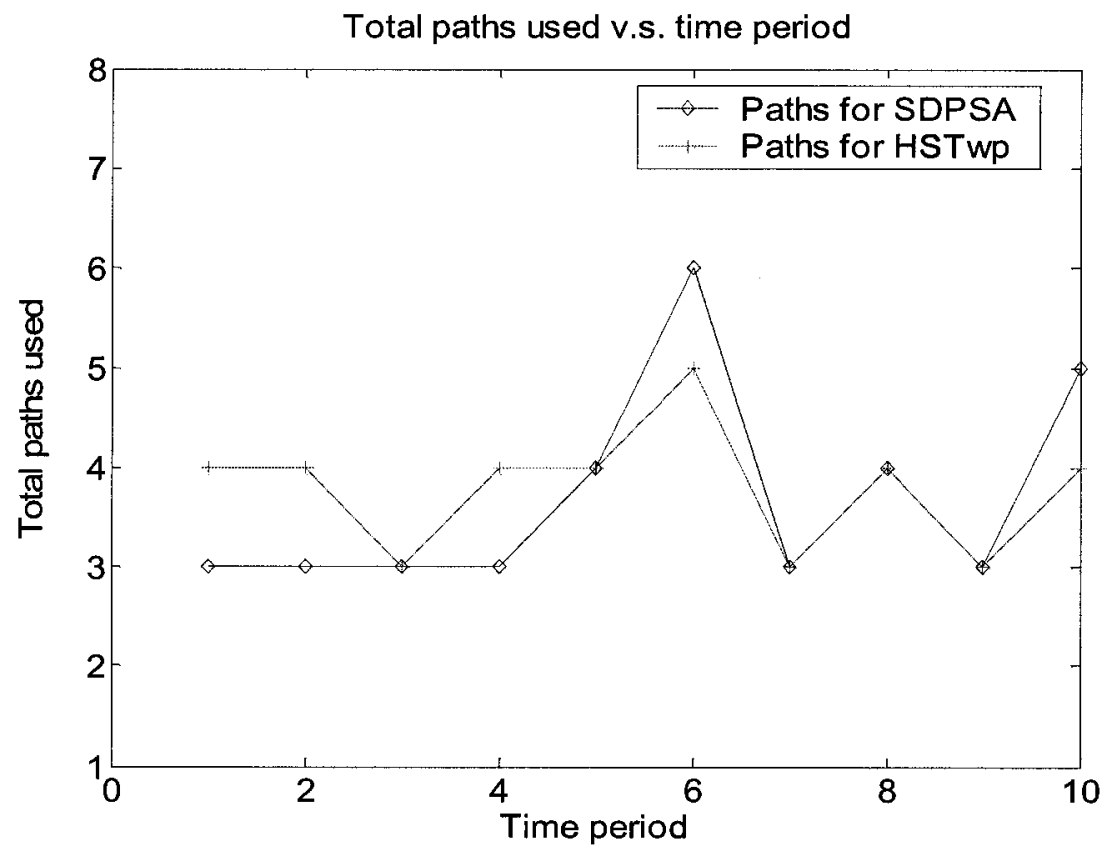

Fig. 5.11 Total paths used v.s. time period for NSFNET

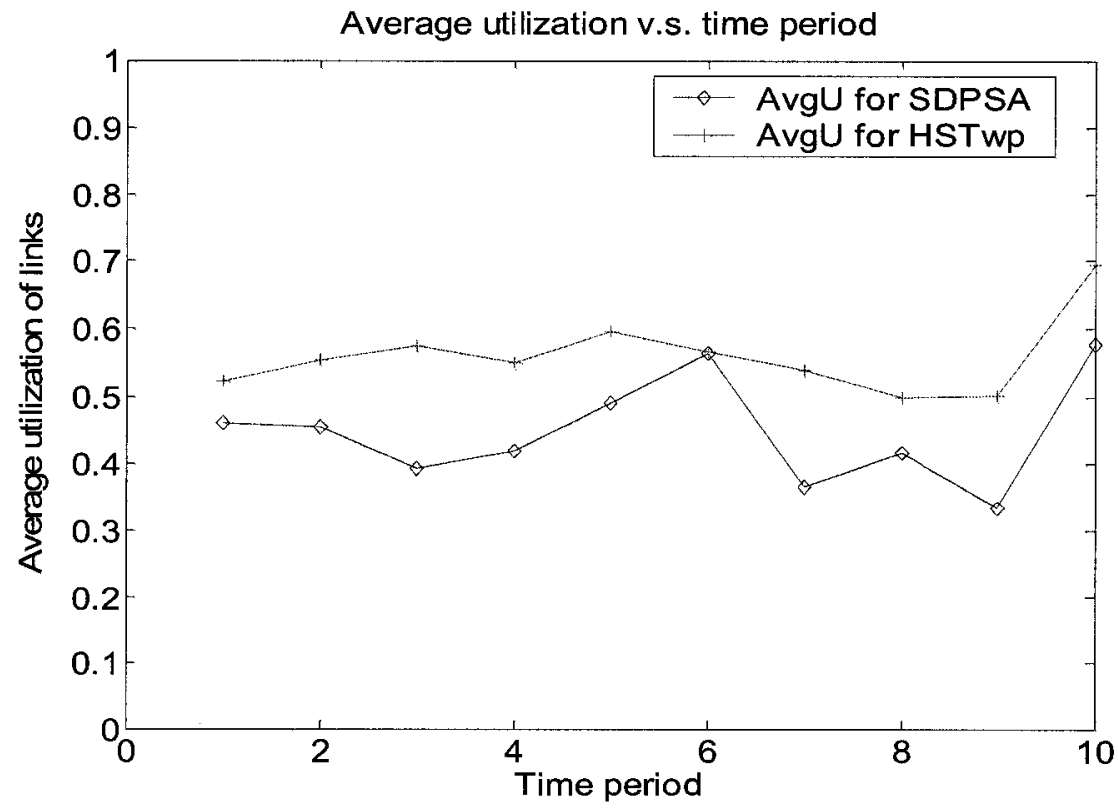

Fig. 5.12 Average utilization among all selected links for NSFNET 
Total hops used v.s. time period

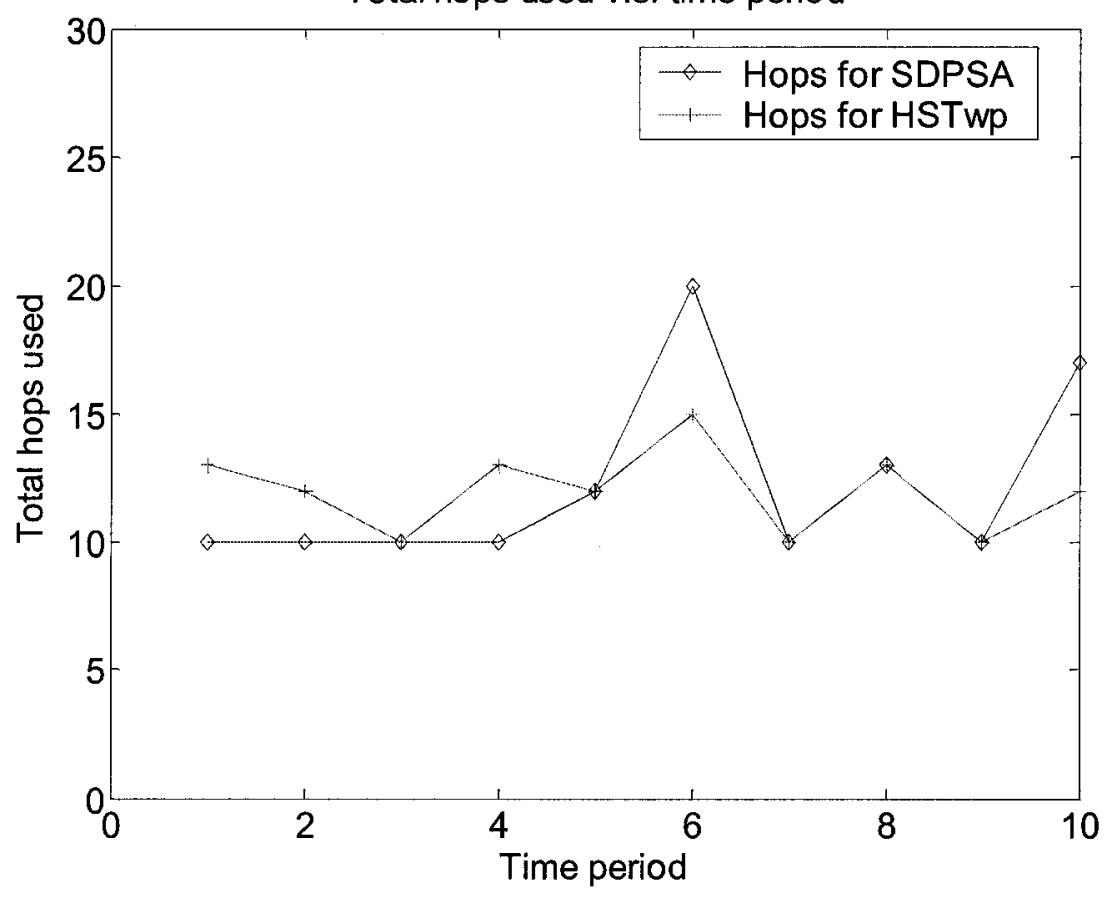

Fig. 5.13 Total hops among all selected paths for NSFNET

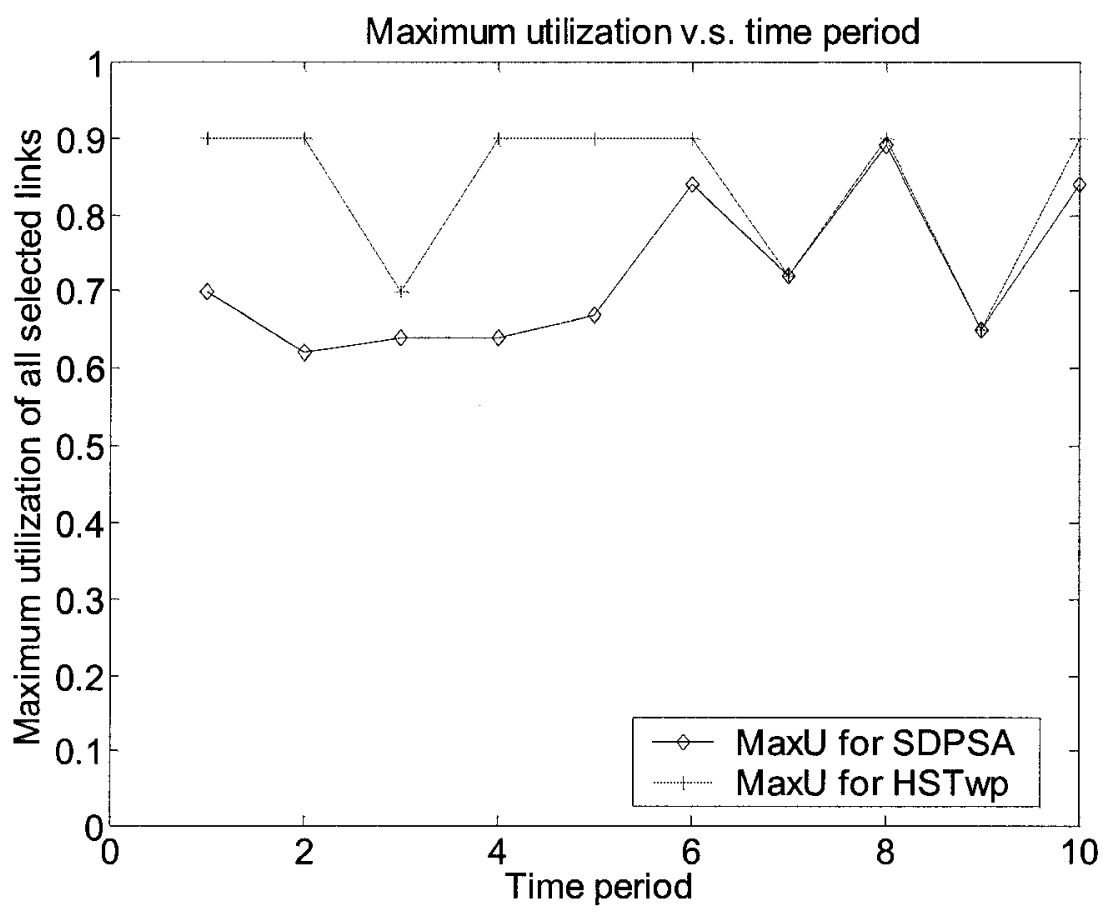

Fig. 5.14 Maximum utilization among all selected links for NSFNET

$-70-$ 


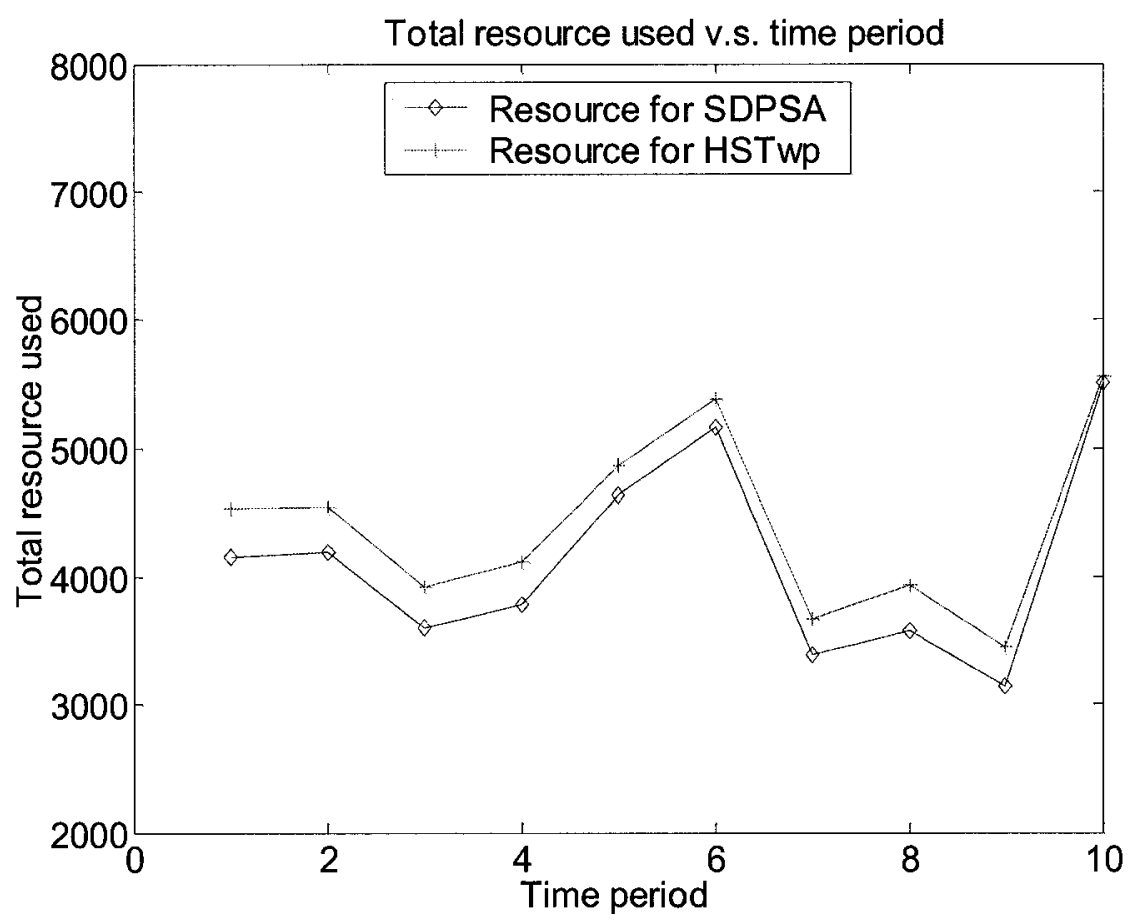

Fig. 5.15 Total resource used among all selected paths for NSFNET

In Fig. 5.11 and Fig. 5.13, paths and hops parameters are compared for these two algorithms throughout various time periods. Based on the results, it is difficult to conclude which one of the two algorithms uses less number of paths or hop counts. The number of hops used is an important parameter when calculating the resource consumption (formula 5.1).

In Fig. 5.12 and Fig. 5.14, average utilization and maximum utilization for all selected links are compared throughout various time periods for the SDPSA algorithm and the HSTwp algorithm. Based on the graphs, it is obvious to see that both the average utilization and the maximum utilization of SDPSA are less than that of HSTwp.

$-71-$ 
Fig. 5.15 and Table 5.2 show that the SDPSA algorithm consistently saves overall network resources over the HSTwp algorithm. On average, by using the SDPSA algorithm, 7.3\% less network resources were consumed comparing to the HSTwp algorithm. The maximum resource saving can reach up to $10 \%$. The overall resource gain for the SDPSA algorithm is less than the gain in scenario I. One of the factors that affect the resource gain is that there exists less disjoint paths for node pairs in NSFNET topology. Nevertheless, our SDPA algorithm does successfully achieve the objective of reducing the total network resource consumption. As a result, by using the SDPSA algorithm, the network can accommodate more traffic demands.

Table 5.2: Total resources consumed on all selected path for NSFNET

\begin{tabular}{|c|c|c|c|}
\hline $\begin{array}{c}\text { Time } \\
\text { period }\end{array}$ & $\begin{array}{c}\text { Resources used in } \\
\text { SDPSA ( }\end{array}$ & $\begin{array}{c}\text { Resources used in } \\
\text { HSTwp }\left(R_{\text {HSTwp }}\right)\end{array}$ & $\begin{array}{c}\text { Resources gain } \\
\left(\left(R_{\text {HSTwp }}-R_{\text {SDPSA }}\right) / R_{\text {SDPSA }}\right)\end{array}$ \\
\hline 1 & 4153 & 4540 & 0.0932 \\
\hline 2 & 4185 & 4546 & 0.0863 \\
\hline 3 & 3608 & 3916 & 0.0854 \\
\hline 4 & 3784 & 4111 & 0.086 \\
\hline 5 & 4632 & 4866 & 0.051 \\
\hline 6 & 5158 & 5371 & 0.042 \\
\hline 7 & 3386 & 3662 & 0.082 \\
\hline 8 & 3576 & 3938 & 0.10 \\
\hline 9 & 3148 & 3454 & 0.0974 \\
\hline 10 & 5500 & 5550 & 0.0091 \\
\hline Average & 4113 & 4395.4 & 0.0732 \\
\hline
\end{tabular}


Table 5.3 shows the number of iterations used in the annealing algorithm in getting the sub-optimal solutions in various time periods. The total network resource usage decreases as the number of iterations increases. By keeping the times of annealing iterations at 3000 , the resulting resources gain can reach about $7.3 \%$. The calculation is less in the SDPSA algorithm because the next period can use the current sub-optimal solution as the initial point. If the initial point can accommodate the next period traffic demands, this initial point (selected path set) will be kept for the next time period.

Table 5.3: Number of times in getting the suboptimal solution

\begin{tabular}{|c|c|c|c|c|c|}
\hline \multirow[b]{2}{*}{ Time period } & HSTwp & \multicolumn{4}{|c|}{ SDPSA } \\
\hline & Total resource used & $\begin{array}{c}1000 \\
\text { times of } \\
\text { annealing } \\
\text { iterations }\end{array}$ & $\begin{array}{c}\text { Total resource } \\
\text { used }\end{array}$ & $\begin{array}{c}3000 \\
\text { times of } \\
\text { annealing } \\
\text { iterations }\end{array}$ & $\begin{array}{c}\text { Total resource } \\
\text { used }\end{array}$ \\
\hline 1 & 4540 & & 4573 & & 4153 \\
\hline 2 & 4546 & & 4555 & & 4185 \\
\hline 3 & 3916 & & 3903 & & 3608 \\
\hline 4 & 4111 & & 4171 & & 3784 \\
\hline 5 & 4866 & & 4919 & & 4632 \\
\hline 6 & 5371 & & 5663 & & 5158 \\
\hline 7 & 3662 & & 3616 & & 3386 \\
\hline 8 & 3938 & & 5035 & & 3576 \\
\hline 9 & 3454 & & 3320 & & 3148 \\
\hline 10 & 5550 & & 5808 & & 5500 \\
\hline
\end{tabular}




\section{Chapter 6}

\section{Conclusions and Future Research}

In this thesis we present a Shortest Distance Path based on Simulated Annealing (SDPSA) algorithm for the multi-path routing and the splittable multi-commodity flow problem for dynamic traffic engineering in backbone networks. The algorithm finds a set of paths with the objective of minimizing the overall network resource consumption and achieving network load balancing at the same time. Moreover, the algorithm takes advantage of the previous sub-optimal solution by using it as the initial point for subsequent annealing process. As a result, the SDPSA algorithm is more efficient in getting new sub-optimal solutions. The SDPSA algorithm is applied to a randomly generated network topology and to the NSFNET topology. After comparing the proposed algorithm with the well-known HSTwp algorithm, the simulation results and comparison analysis show that the SDPSA algorithm has a superior performance over the HSTwp algorithm in terms of network resource consumption. Furthermore, in accommodating the dynamic traffic demands, the SDPSA algorithm is more scalable because of its fast and efficient path selection and traffic assignment. 
By using the multi-path routing with bandwidth reservation over backbone networks, the SDPSA algorithm gives a sub-optimal solution regarding the dynamic traffic demands. The simulated annealing method used in the algorithm takes advantage of previous multipath routing information as its initial point for the current period. This is an efficient way to obtain multi-path routing solutions with dynamic traffic demands.

It is necessary for future research to uncover the applicability of the SDPSA algorithm to different applications such as VoIP and VPN. Improving the algorithm, for example, in regards to the alternative stopping conditions in the algorithm could be possible direction for future research. 


\section{References}

[1] R. Braden, D. Clark, and S. Shenker, "Integrated Services in the Internet Architecture: an Overview", Internet RFC 1633, June 1994

[2] S. Blake, D. Black, M. Carlson et al., "An Architecture for Differentiated Services", Internet RFC2475, 1998

[3] E. Rosen, A. Viswanathan, and R. Callon, "Multiprotocol Label Switching", Internet RFC3031, http://www.ietf.org/rfc/rfc3031.txt.

[4] J. Moy, “OSPF Version 2”, RFC 2178, 1997

[5] Q. Ma and P. Steenkiste, "On Path Selection for Traffic with Bandwidth Guarantees", Network Protocols, 1997. Proceedings., 1997 International Conference on 28-31 Oct. 1997 Page(s):191 - 202

[6] Q. Ma and P. Steenkiste, "Routing Traffic with Quality-of-Service Guarantees in Integrated Services Networks", In $8^{\text {th }}$ IEEE/ACM International Workshop on Network and Operating Systems Support for Digital Audio and video, England, July 1998.

[7] G. Kesidis, J. Walrand, C.-S. Chang, "Effective bandwidths for multi-class Markov fluids and other ATM sources", Networking, IEEE/ACM Transactions on , Volume: 1 Issue: 4 , Aug. 1993 Page(s): 424 -428

[8] R. Boorstyn, A. Burchard, J. Liebeherr, C. Oottamakorn, "Effective envelopes: statistical bounds on multiplexed traffic in packet networks", INFOCOM 2000 
Nineteenth Annual Joint Conference of the IEEE Computer and Communications Societies, Proceedings IEEE, Volume: 3 , 26-30 March 2000 Page(s): 1223 1232 vol.3

[9] C. Huang, I. Lambadaris, M. Devetsikiotis, P. W. Glynn, and A. R. Kaye, "DTMW: A New Congestion Control Scheme for Long-Range Dependent Traffic", presented at the 15th International Teletraffic Congress, ITC 15, Washington, D.C., June 1997.

[10]F. Kelly, "Notes on effective bandwidths," Stochastic Networks: Theory and Applications, ClarendonPress, Oxford, 1996.

[11]K.M. Rege, "Equivalent bandwidth and related admission criteria ATM systems-a performance study," International Journal of Communication Systems, vol. 7. pp.181-197, 1994

[12]Z.L. Zhang, Z. Liu, J. Kurose and D. Towsley, "Call Admission Control Schemes Under Generalized Processor Sharing Scheduling", Telecommunication Systems 7, pp.125-152, 1997

[13]S. Tartarelli, M. Falkner, M. Devetsikiotis, I. Lambadaris, S. Giordano, "Empirical effective bandwidths", Global Telecommunications Conference, 2000. (GLOBECOM'00 IEEE), vol.1, pp. $672-678,2000$

[14]H. Li, C. Huang, M. Devetsikiotis and G. Damm, "Effective Bandwidths under Dynamic Weighted Round Robin Scheduling”, Accepted by 47th annual IEEE Global Telecommunications Conference (GLOBECOM 2004), Dallas, Texas, USA, 29 Nov. - 3 Dec. 2004. 
[15]A.Elwalid, C.Jin, S.Low and I.Widjaja, "MATE: MPLS Adaptive Traffic Engineering”, INFOCOM 2001. Twentieth Annual Joint Conference of the IEEE Computer and Communications Societies. Proceedings. IEEE,Vol.3, 2001,pp.1300-1309.

[16] S.D. Patek, R. Venkateswaran, J. Liebeherr, "Enhancing aggregate QoS through alternate routing", Global Telecommunications Conference, 2000. GLOBECOM '00.IEEE Volume 1, 27 Nov.-1 Dec. 2000 Page(s):611 - 615 vol.1

[17]K. Papagiannaki, N. Taft, Z. Zhang \& C. Diot, "Long-Term Forecasting of Internet Backbone Traffic-Observations and Initial Models", Conf. Computer Communications, IEEE INFOCOM, 2003

[18] A.W. Berger, Y. Kogan, "Dimensioning bandwidth for elastic traffic in highspeed data networks", Networking, IEEE/ACM Transactions on, Oct. 2000.

[19]B. Fortz and M. Thorup, "Optimizing OSPF/IS-IS Weights in a Changing World," IEEE Journal on Selected Areas in Communications (JSAC), 20(4):756-766, May 2002.

[20]Y. Wang, and Z. Wang, "Explicit routing algorithms for Internet traffic engineering", Computer Communications and Networks, 1999. Proceedings. Eight International Conference on 11-13 Oct. 1999 Page(s):582 - 588

[21]M. Kodialam and T.V. Lakshman, "Minimum Interference Routing with Applications to MPLS Traffic Engineering", INFOCOM 2000. Nineteenth Annual Joint Conference of the IEEE Computer and Communications Societies. Proceedings. IEEE Volume 2, 26-30 March 2000 Page(s):884 - 893 vol.2 
[22]Y. Lee, Y. Seok, and Y. Choi, "Traffic Engineering with constrained multi-path routing in MPLS networks", Communications, 2002. ICC 2002. IEEE International Conference on Volume 4, 28 April-2 May 2002 Page(s):2431 2436 vol.4

[23]C. Villamizar and T. Li, "IS-IS Optimized Multipath (IS-IS OMP)", Internet draft $<$ draft-villamizar-isisomp-00.txt>, Oct. 1998

[24] X. Xiao and L.M Ni, "Internet QoS: a big picture", Network, IEEE , Volume: 13 , Issue: 2 , March-April 1999 Pages:8 - 18

[25]S. Jordan, "Pricing of Buffer and Bandwidth in a Reservation-Based QoS Architecture", IEEE International Conference on Communications, Ancourage, Alaska, May 2003, pp.1521-1525

[26]R.J. Gibbens, F.P. Kelly, "Resource pricing and the evolution of congestion control", Automatica, 35 (1999), 1969-1985

[27]J.K. MacKie-Mason, H.R. Varian, "Pricing the Internet", in B. Kahin and J. Keller, eds., Public Access to the Internet, MIT Press, Cambridge, MA, 1995.

[28]E.W. Fulp, D.S. Reeves, "Optimal provisioning and pricing of Internet differentiated services in hierarchical markets". First International Conference Colmar, France, July 9-13, 2001, Proceedings, Part 1.

[29]R. Guerin, D.Williams, and A. Orda, "QoS routing mechanisms and OSPF extensions", GLOBECOM, Nov. 1997 IEEE , Volume: 3 , 3-8 Nov. 1997

[30]A. Shaikh, J. Rexford, and K. Shin, "Evaluating the impact of stale link state on quality of service routing," IEEE Trans. Networking, vol. 9. pp.162-176, Apr, 2001. 
[31] G. Apostolopoulos, R. Guerin, S. Kamat, S. Tripathi, "Quality of Service Based Routing: A Performance Perspective”, ACM SIGCOMM 1998.

[32] Q. Ma, "QoS Routing in the Integrated Services networks", Ph.D. thesis, CMUCS-98-138, Jan. 1998

[33] S. Nelakuditi, Z.L. Zhang, R.P. Tsang, H.C, Du, “Adaptive proportional routing: a localized QoS routing approach", Networking, IEEE/ACM Transactions on ,Volume: 10 Issue: 6 , Dec. 2002 Page(s): 790 -804

[34] S. Nelakuditi, R.P. Tsang, Z.L. Zhang, "Quality of service routing without global information exchange", Quality of Service, 1999. IWQoS '99, 1999 Seventh International Workshop on, 31 May-4 June 1999 Page(s): 129 -131

[35]M. Shi, S. Wang, Y. Bai, "A bandwidth constrained QoS routing optimization algorithm", Communication Technology Proceedings, 2003. ICCT 2003 International Conference on, Volume: 1, April, 2003.

[36]R.F. Farmer and I. Kaufman, "On the Numerical Evaluation of Some Basic Traffic Formulae," in Networks, Vol 8., pp. 153-186, John Wiley and Sons, Inc., 1978.

[37]B. Fortz and M. Thorup, "Internet Traffic Engineering by Optimizing OSPF Weights", Proceedings of the INFOCOM 2000, pp. 519-528, 2000

[38]Z. Wang, J. Crowcroft, "Quality-of-service routing for supporting multimedia applications", Selected Areas in Communications, IEEE Journal on , Volume: 14 Issue: 7 , Sept. 1996 Page(s): $1228-1234$

[39] R. Ahuja, T. Magnanti, and J. Orlin, "Network Flows". Prentice Hall, 1993 
[40] S. Suri, M. Waldvogel, D. Bauer \& P. R. Warkhede, "Profile based routing and traffic engineering", Computer Communications, vol. 26, 2003.

[41]J.C. Spall, “ Introduction to Stochastic Search and Optimization: Estimation, Simulation, and Control", Wiley, Hoboken, NJ, 2003

[42] Heaton Research, "Programming Neural Networks in Java", http://www.heatonresearch.com/

[43] A. Medina, A. Lakhina, I. Matta, and J. Byers, "Brite: An approach to universal topology generation", Proc. MASCOTS Aug, 2001

[44] J.Y. Yen, "Finding the K shortest loop-less paths in a network", Management Science 17:712-716, 1971

[45] J.E. Hershberger, M. Maxel, and S. Suri, "Algorithm Engineering \& Experiments (ALENEX)", Proc. 5th Worksh. SIAM, Jan 2003. 


\section{Appendix}

\section{The source code for Obtaining Candidate Path Set for Given Topology -NSFNET}

The source code for calculating the candidate path set for given NSFNET topology is given below and the source code is programmed in C. The input is the adjacent matrixMatrix $[i][j]$ (where $i$ and $j$ are the node in NSFNET) for NSFNET topology and the output is the candidate path set for each pair of nodes in path.txt file. See topology in Fig. 5.9 .

\#include <iostream.h $>$

\#include $<$ stdio.h $>$

\#define NumNode 14

int Matrix[NumNode][NumNode];

int NoOfPaths;

FILE * f;

void printPath(int path[NumNode], int node)

\{

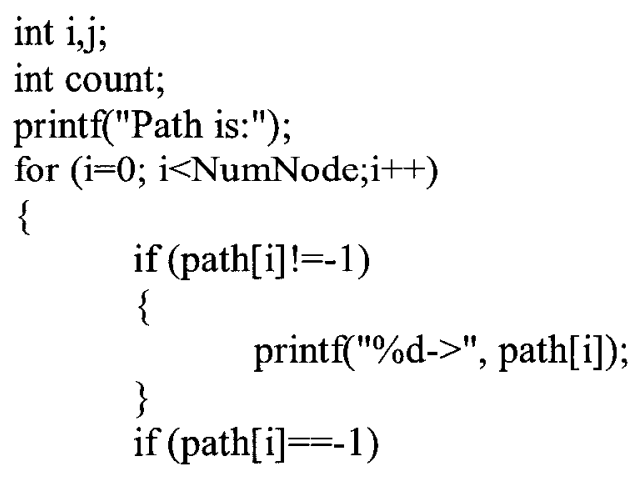




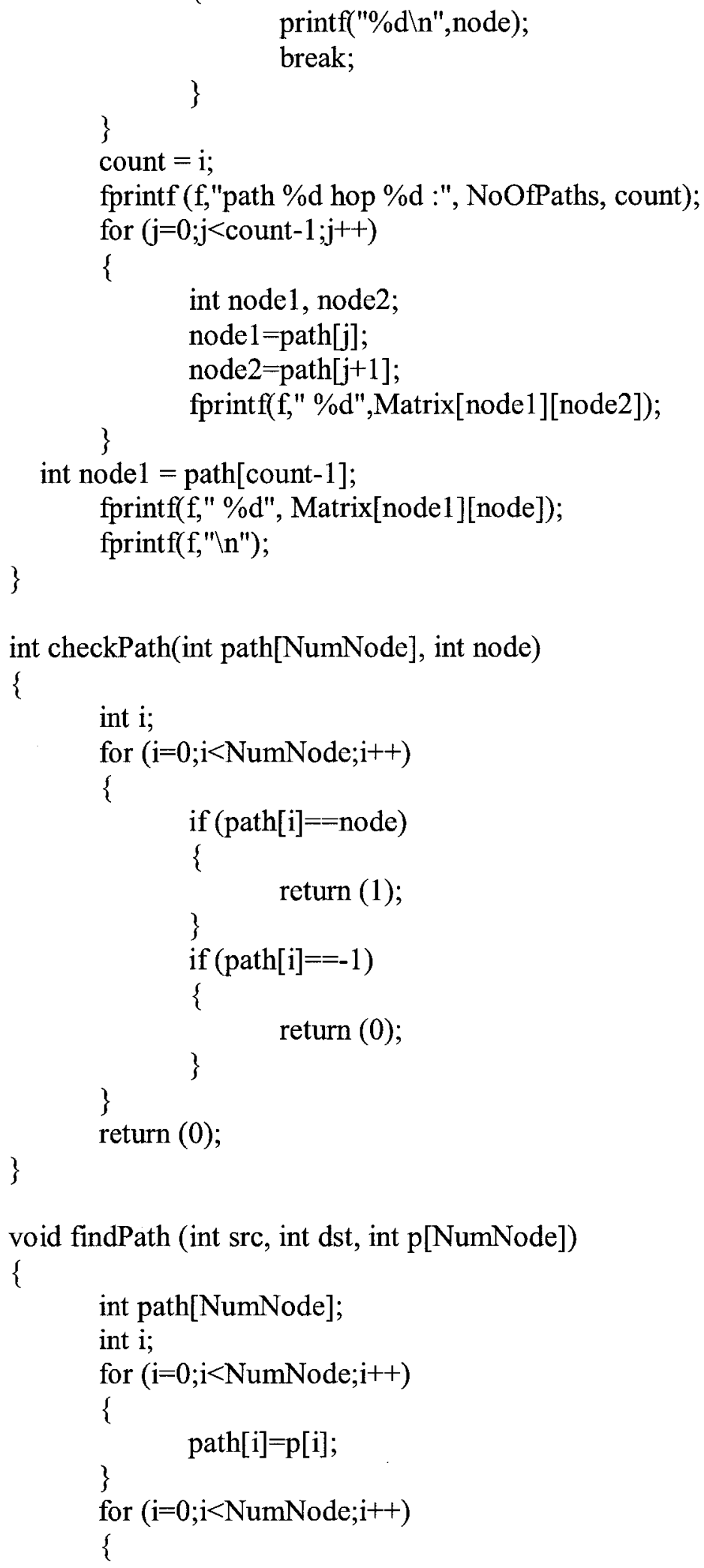




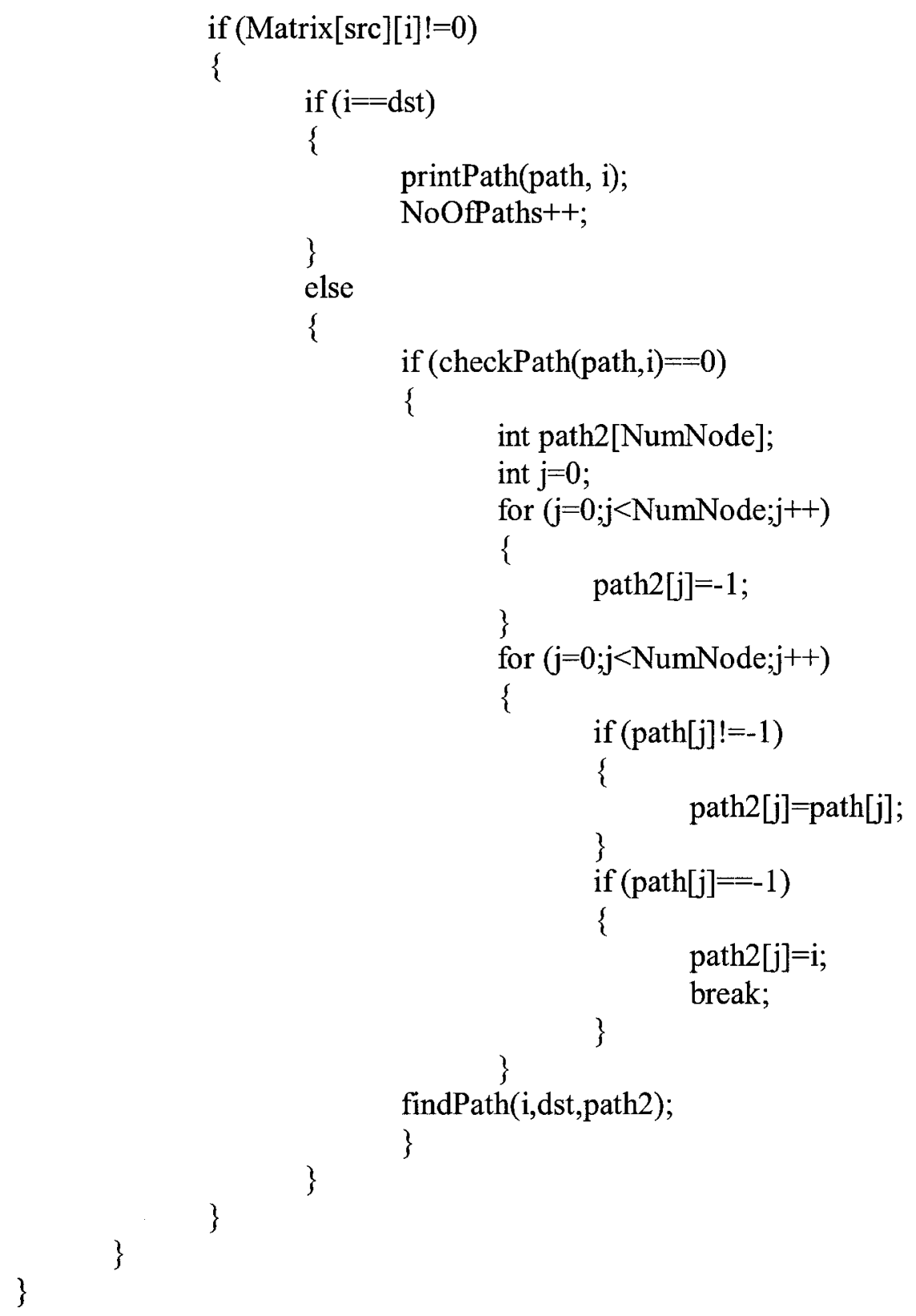

void main ()

\{

$f=$ fopen ("path.txt", "w");

int src, dst;

int $\mathrm{p}$ [NumNode];

int $\mathrm{i}, \mathrm{j}$;

for $(\mathrm{i}=0 ; \mathrm{i}<$ NumNode $; \mathrm{i}++)$

\{

- 84 - 


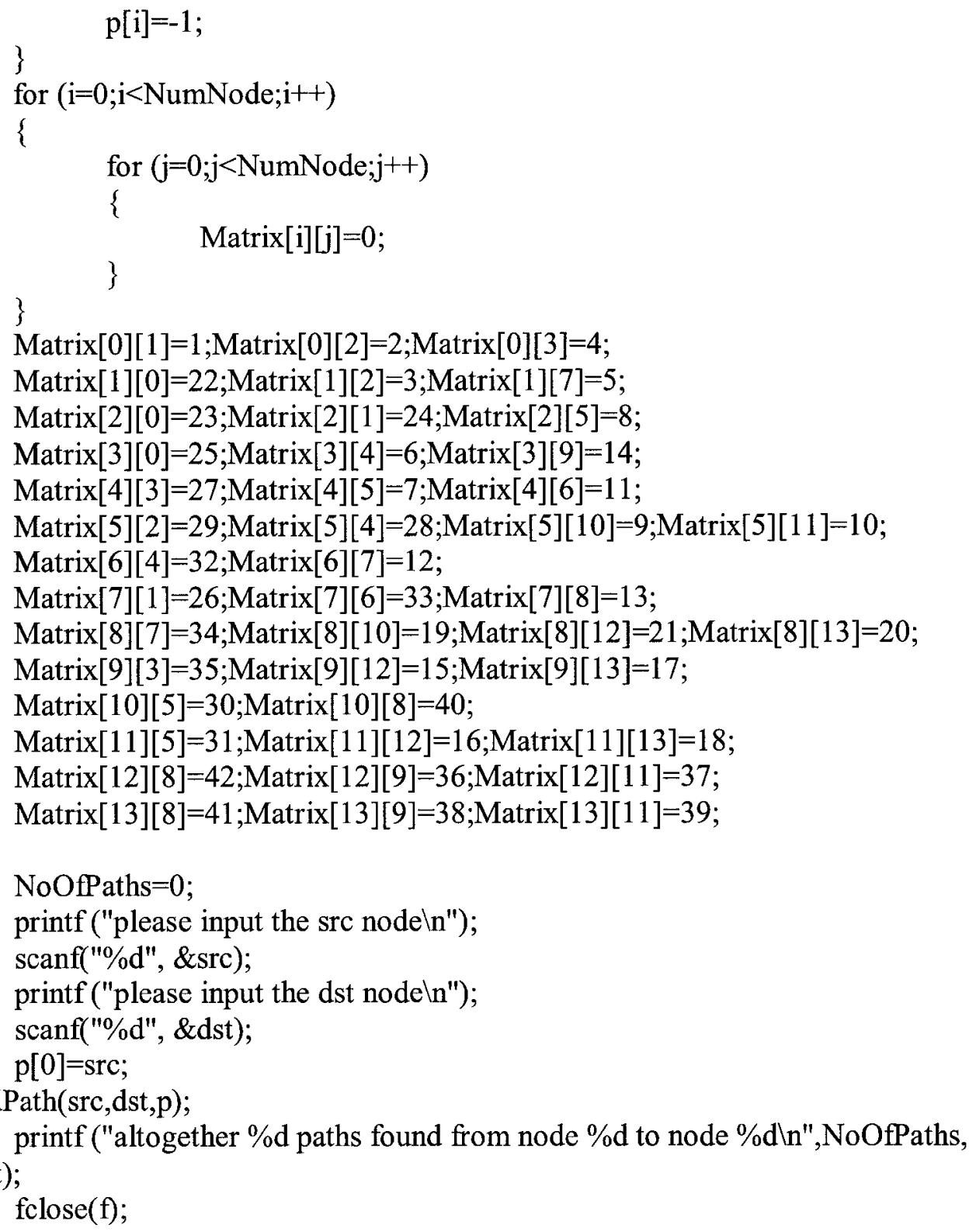

